\title{
Diagenetic evolution of fault zones in Urgonian microporous carbonates, impact on reservoir properties (Provence - southeast France)
}

\author{
Irène Aubert, Philippe Léonide, Juliette Lamarche, and Roland Salardon \\ Aix-Marseille Université, CNRS, IRD, Cerege, Um 34, 3 Place Victor Hugo (Case 67), 13331 Marseille Cedex 03, France
}

Correspondence: Irène Aubert (aubertirene@gmail.com)

Received: 8 October 2019 - Discussion started: 28 October 2019

Revised: 8 April 2020 - Accepted: 2 May 2020 - Published: 5 July 2020

\begin{abstract}
Microporous carbonate rocks form important reservoirs with permeability variability depending on sedimentary, structural, and diagenetic factors. Carbonates are very sensitive to fluid-rock interactions that lead to secondary diagenetic processes like cementation and dissolution capable of modifying the reservoir properties. Focusing on fault-related diagenesis, the aim of this study is to identify the impact of the fault zone on reservoir quality. This contribution focuses on two fault zones east of La Fare anticline (SE France) crosscutting Urgonian microporous carbonates. Overall, 122 collected samples along four transects orthogonal to fault strike were analyzed. Porosity values have been measured on 92 dry plugs. Diagenetic elements were determined through the observation of 92 thin sections using polarized light microscopy, cathodoluminescence, carbonate staining, SEM, and stable isotopic measurements $\left(\delta^{13} \mathrm{C}\right.$ and $\left.\delta^{18} \mathrm{O}\right)$. Eight different calcite cementation stages and two micrite micro-fabrics were identified. As a main result, this study highlights that the two fault zones acted as drains canalizing low-temperature fluids at their onset and induced calcite cementation, which strongly altered and modified the local reservoir properties.
\end{abstract}

\section{Introduction}

Microporous carbonates form important reservoirs (Deville de Periere et al., 2017; Lambert et al., 2006; Sallier, 2005; Volery et al., 2009), with porosity values up to $35 \%$ (Deville de Periere et al., 2011). Due to their heterogeneous properties, which depend on sedimentary, structural, and diagenetic factors, microporous carbonates may determine a high variability in reservoir permeability (Bruna et al., 2015; Deville de Periere et al., 2011, 2017; Eltom et al., 2018; Florida et al., 2009; Hollis et al., 2010). Moreover, fault zones in carbonates play an important role on reservoir properties (Agosta et al., 2010, 2012; Caine et al., 1996; Delle Piane et al., 2016; Ferraro et al., 2019; Knipe, 1993; Laubach et al., 2010; Rossetti et al., 2011; Sinisi et al., 2016; Solum et al., 2010; Solum and Huisman, 2016; Tondi, 2007; Wu et al., 2019). Fault zones are complex structures composed of damage zones and the fault core encompassed by the host rock (Caine et al., 1996; Chester and Logan, 1986, 1987; Hammond and Evans, 2003). Faults can act as barriers (Agosta et al., 2010; Tondi, 2007), drains (Agosta et al., 2007, 2008, 2012; Delle Piane et al., 2016; Evans et al., 1997; Molli et al., 2010; Reches and Dewers, 2005; Sinisi et al., 2016; Solum and Huisman, 2016), or mixed hydraulic behavior zones (Matonti et al., 2012) depending on their architecture and diagenetic evolution. Because of their hydraulic properties, fault zones influence the fluid flows in the upper part of Earth's crust (Bense et al., 2013; Evans et al., 1997; Knipe, 1993; Sibson, 1994; Zhang et al., 2008) and are capable of increasing the fluidrock interactions. Carbonates are very sensitive to these interactions, which lead to diagenetic secondary processes like cementation and dissolution (Deville de Periere et al., 2017; Fournier and Borgomano, 2009; Lambert et al., 2006). Faultrelated diagenesis locally modifies the initial rock properties (mineralogy and porosity) and therefore the reservoir properties (Hodson et al., 2016; Knipe, 1993; Knipe et al., 1998; Laubach et al., 2010; Woodcock et al., 2007). In case of a polyphasic fault zone, repeating fluid pathways-barriers behavior in time leads to very complex diagenetic modifica- 
tions. The initial vertical and lateral compartmentalization of microporous limestones is, therefore, accentuated by faultrelated diagenesis. Hence, understanding faulting and diagenesis processes is crucial for a better exploration and production in carbonates. Urgonian microporous carbonates of Provence are made of facies and reservoir properties analogue to Middle Eastern microporous carbonate reservoirs (Thamama, Kharaib, and Shuaiba Formations; Borgomano et al., 2002, 2013; Sallier 2005; Fournier et al., 2011; Léonide et al., 2012, 2014). Although Urgonian microporous carbonates of Provence are analogue to Middle Eastern reservoirs, the analogy can be extended to other faulted microporous carbonate reservoirs. To have a better comprehension of diagenetic modifications linked to fault zones in these rocks, the aim of this paper is (i) to determine the diagenetic evolution of polyphasic fault zones, (ii) to identify their impact on reservoir properties, and (iii) to link the fault evolution with the fluid flow and geodynamic history of the basin.

\section{Geological context}

We studied two faults crosscutting microporous Valanginianto-Early Aptian Urgonian carbonates of the Southeast Basin (Provence - SE France) deposited along the southern margin of the Vocontian Basin (Léonide et al., 2014; Masse and Fenerci Masse, 2011). The "Urgonian" platform carbonates (Masse, 1976) reached their maximum areal extension during the late Hauterivian-Early Aptian (Masse and Fenerci-Masse, 2006). From Albian to Cenomanian, the regional Durancian uplift triggered exhumation of Early Cretaceous carbonates, bauxitic deposition (Guyonnet-Benaize et al., 2010; Lavenu et al., 2013; Léonide et al., 2014; Masse and Philip, 1976; Masse, 1976), and development of E-Wtrending extensional faults (Guyonnet-Benaize et al., 2010; Masse and Philip, 1976). During Late Cretaceous times, platform environment led to a transgressive rudist deposition (Philip, 1970). From Late Cretaceous to Eocene, the convergence between Iberia and Eurasia plates (e.g., Bestani 2015, and references therein) caused a regional N-S shortening (e.g., Molliex et al., 2011 and references therein). The so-called "Pyrénéo-Provençal" shortening, gave rise to E-W-trending, north-verging thrust faults and ramp folds (e.g., Bestani et al., 2016, and references therein). From Oligocene to Miocene, the area underwent extension associated with Liguro-Provençal Basin opening (e.g., Demory et al., 2011). During Mio-Pliocene times, the Alpine shortening dimly impacted the studied area (Besson, 2005; Bestani, 2015) and reactivated the "Pyrénéo-Provençal" structures (Champion et al., 2000; Molliex et al., 2011).

We studied two faults pertaining to a kilometer-scale fault system on the E-W-trending La Fare anticline near Marseille (Fig. 1A). The southern limb of this anticline dips $25^{\circ} \mathrm{S}$ and is constituted by Upper Hauterivian, Lower Barremian, and Santonian rocks (Fig. 1B). The Upper Barremian carbon- ates are composed, from bottom to top, of a $120 \mathrm{~m}$ thick calcarenitic unit with cross-beddings, a $40 \mathrm{~m}$ thick massive coral-rich calcarenite unit, and an upper $10 \mathrm{~m}$ thick calcarenite unit (Masse, 1976; Matonti et al., 2012; Roche, 2008). Santonian-age coarse rudist limestones uncomfortably overlap the Barremian carbonates (Fig. 1A).

The Castellas Fault zone is a $2.14 \mathrm{~km}$ long left-lateral strike-slip fault, 60 to $70^{\circ} \mathrm{N}$ trending and 40 to $80^{\circ} \mathrm{N}$ dipping (Fig. 2A, B; Table 1) composed of horse structures, secondary faults, and lenses (Fig. 2A, C; Aubert et al., 2019b). The second investigated fault zone "D19" is composed of five sub-fault zones (F1 to F5) restricted in a $50 \mathrm{~m}$ long interval (Fig. 2E, H; Table 1; Aubert et al., 2019a). Sub-faults are organized into two sets. The first one comprises F3 and F4, 40 to $55^{\circ} \mathrm{N}$ trending, $60-80^{\circ} \mathrm{NW}$ dipping (orange traces in Fig. 2F). Set 2 is $30^{\circ} \mathrm{N}$ trending, dipping $80^{\circ} \mathrm{E}$, with leftlateral strike-slip slickensides pitch 20 to $28^{\circ} \mathrm{SW}$ (F1, F2, F5, red traces in Fig. 2F).

The internal structure of both fault zones results from three distinct tectonic events:

- the Durancian uplift dated as mid-Cretaceous leading to extension and to normal en échelon normal faults; the Castellas Fault nucleated during this first extensional event and bears early dip-slip normal striations (Matonti et al., 2012);

- the Early Pyrenean compression with 0 to $170^{\circ} \mathrm{N}$ trending $\sigma_{1}$ (see cited references in Espurt et al., 2012), which reactivated the Castellas Fault as sinistral (Matonti et al., 2012) and led to the newly formed strike-slip faults of the D19 outcrop (Aubert et al., 2019a);

- the Pyrenean to Alpine folding, triggering the $25^{\circ} \mathrm{S}$ tilting of the strata and fault zones; faults of the D19 outcrop were reactivated, while the Castellas Fault tilting led to an apparent present-day reverse throw (Aubert et al., 2019a).

\section{Database}

We performed four transects across the Castellas Fault and the D19 Fault zone (Fig. 2). Transect 1 is located along the coral-rich unit 2. This lithostratigraphic unit is essentially composed of peloidal grains and bioclasts (corals, bivalves, and stromatoporidae; Fig. 2Da). Transects 2 and 3 crosscut unit 3, made of fine calcarenites with peloidal grains and a rich fauna (foraminifera, bivalves, ostracods, and echinoderms; Fig. 2Db, c). Transect 4 was conducted along the D19 outcrop (Fig. 3), which exposes Barremian outer-platform bioclastic calcarenite with current ripples. The grains are mainly peloids with minor amounts of bioclasts (solidary corals, bryozoans, bivalves, and some rare miliolids; Fig. 2Ga). 

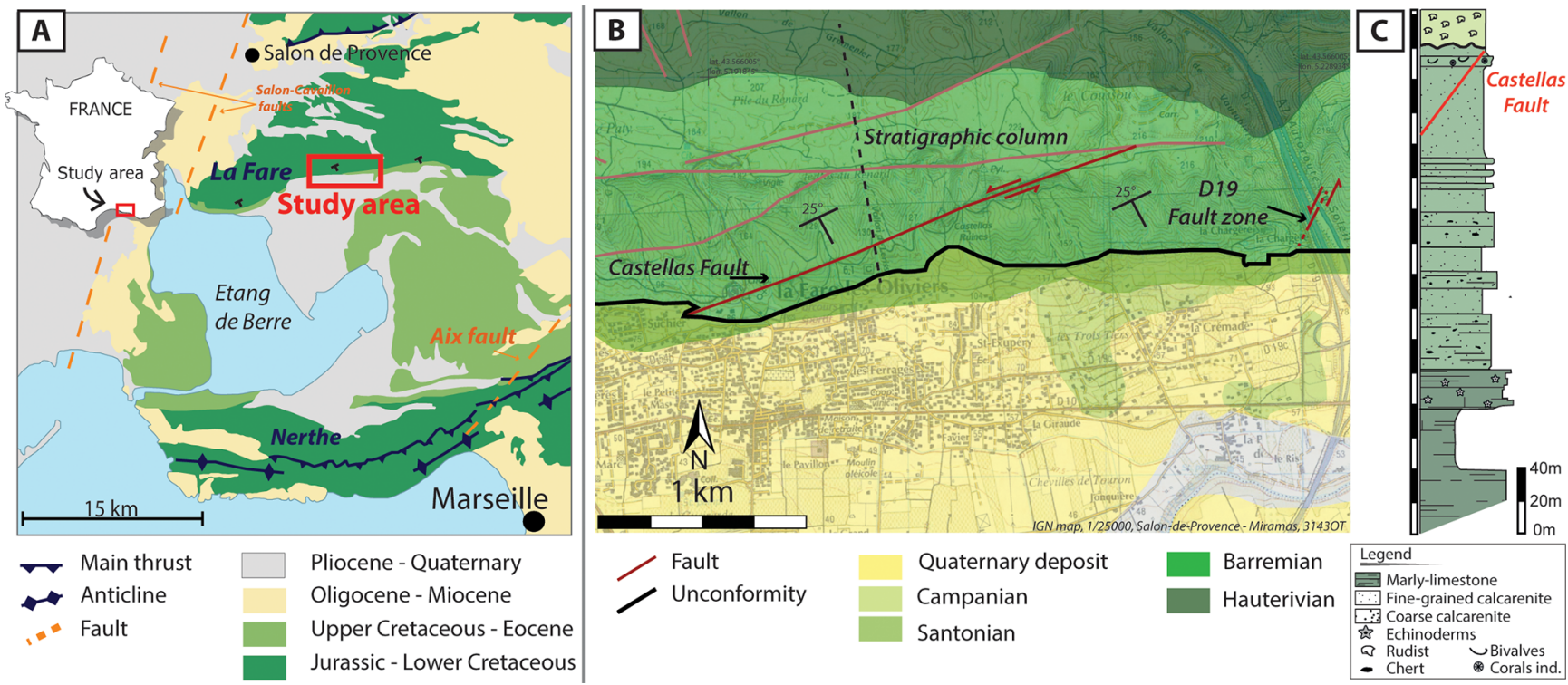

Figure 1. Geological context of the study area. (A) geological map of Provence, (B) Simplified structural map with the location of the Castellas Fault and the stratigraphic column (black dashed line); (C) Stratigraphic column of exposed Cretaceous carbonates (modified from Roche, 2008).

Table 1. Structural properties of the fault zones.

\begin{tabular}{|c|c|c|c|c|c|c|c|c|c|}
\hline \multirow{2}{*}{$\begin{array}{l}\text { Fault } \\
\text { zones }\end{array}$} & \multirow[t]{2}{*}{ Fault } & \multirow[t]{2}{*}{ Direction } & \multirow[t]{2}{*}{ Dip } & \multirow{2}{*}{$\begin{array}{l}\text { Dip } \\
\text { direction }\end{array}$} & \multirow{2}{*}{$\begin{array}{l}\text { Pitch } \\
\text { striation }\end{array}$} & \multirow{2}{*}{$\begin{array}{l}\text { Fault core } \\
\text { thickness }\end{array}$} & \multicolumn{3}{|c|}{ Fault rocks } \\
\hline & & & & & & & FR1 & FR2 & FR3 \\
\hline Castellas & Castellas & $60-70$ & $40-80$ & $\mathrm{~N}$ & $14 \mathrm{~W}-$ & 0 to $4 \mathrm{~m}$ & sparsely present & majoritarily present & / \\
\hline \multirow[t]{5}{*}{ D19 } & $\mathrm{F} 1$ & 30 & 56 & $\mathrm{~W}$ & & 20 & I & $<10 \mathrm{~cm}$ & I \\
\hline & $\mathrm{F} 2$ & 29 & 70 & $\mathrm{E}$ & $28 \mathrm{~S}$ & 10 to 15 & I & $?$ & variable thickness \\
\hline & F3 & 56 & 80 & $\mathrm{~N}$ & & 0 to 15 & l & $?$ & $?$ \\
\hline & $\mathrm{F} 4$ & 42 & 70 & W & & 20 & I & in clasts of FR3 & variable thickness \\
\hline & F5 & 32 & 85 & $\mathrm{~N}$ & $20 \mathrm{SW}$ & 50 to 100 & l & l & variable thickness \\
\hline
\end{tabular}

The different tectonic events impacted the fault zone and fault core structure. Three different fault rock types were identified in the fault core of the two investigated fault zones (see Aubert et al., 2019a; Matonti et al., 2012). Fault rock 1 (FR1) results from the extensional activation of the Castellas Fault during Durancian uplift. It is a cohesive breccia composed of subrounded to rounded clasts from the nearby damage zone and $<30 \%$ of fine-grey matrix (Fig. 2Dd). Fault rock 2 (FR2) is linked to the strike-slip reactivation of the Castellas Fault and to the onset of the D19 Fault zone during the Pyrenean shortening. FR2 presents two morphologies depending on the fault zones. Within Castellas Fault, FR2 is an un-cohesive breccia with an orange/oxidized matrix with angular to subrounded clasts belonging to the nearby damage zone and from FR1 (Fig. 2Dd). In the D19 Fault zone, FR2 is a cohesive breccia with rounded clasts of the damage zone and a white cemented matrix (Fig. 2Gb). Fault rock 3 (FR3) is formed by the reactivation of the D19 Fault zone. The timing of D19 Fault reactivation is tricky to determine as it can be related both to Pyrenean or Alpine shortening. FR3 is composed of angular to subangular clasts from FR2 and from the nearby damage zone dispersed in an orange/oxidized matrix $(<20 \%)$ (Fig. 2Gb).

\section{Methods}

The data set comprises 122 samples, 62 from Castellas and 60 from D19 outcrops, collected along the four transects. Porosity values were measured on 92 dry plugs with a Micromeritics AccuPyc 1330 helium pycnometer. Microfacies were determined on 92 thin sections. Impregnation with a blue-epoxy resin allowed us to decipher the different pore types. Thin sections were colored with a solution of hydrochloric acid, Alizarin Red S, and potassium ferricyanide to distinguish carbonate minerals (calcite and dolomite). Thin sections were analyzed using cathodoluminescence to discriminate the different generations of calcite cements. The paragenetic sequence was defined based on 

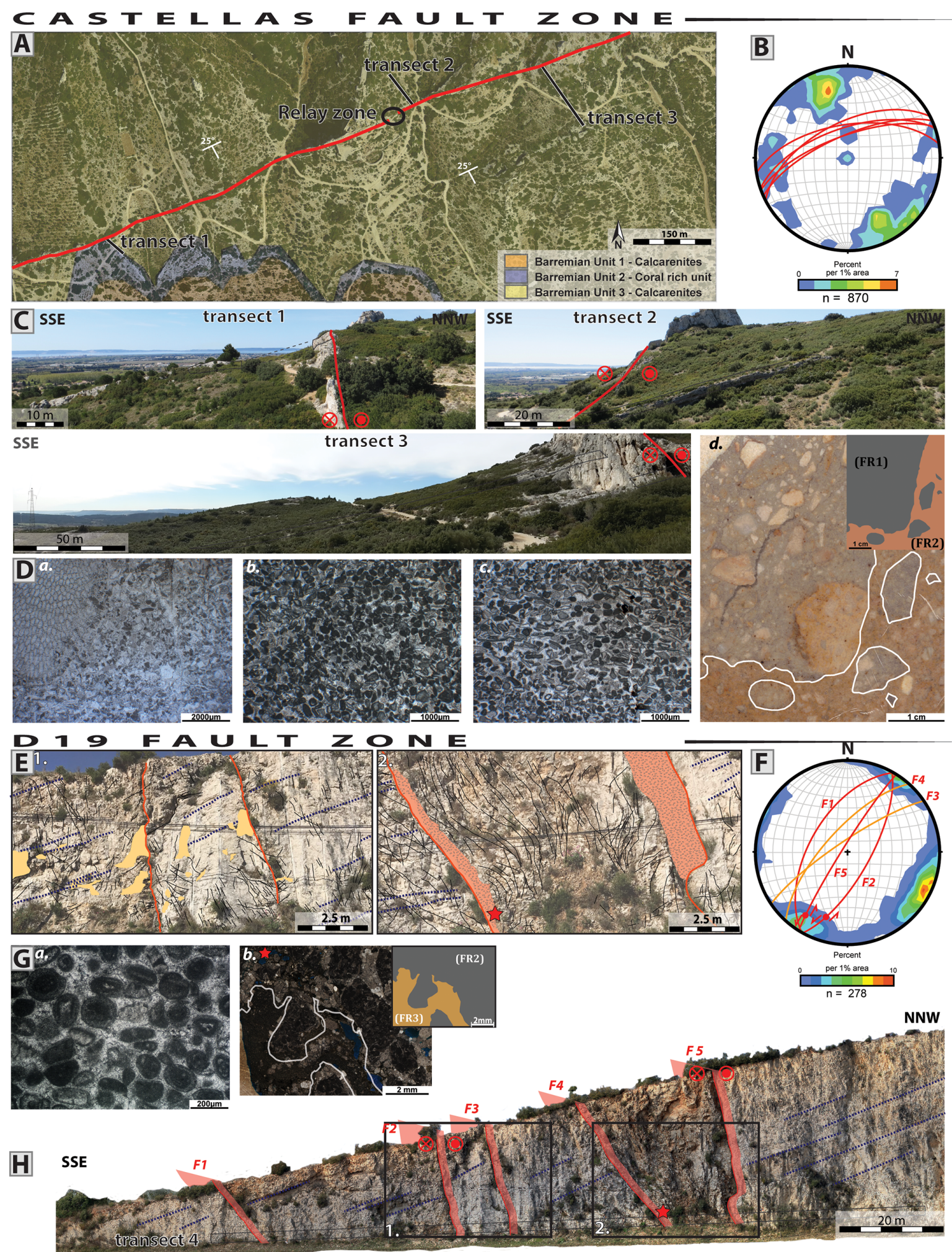

Figure 2. (A) Castellas Fault map on aerial photo with position of the studied transects and the relay zone; (B) stereographic projection of poles to fractures (density contoured) and faults (red lines) (Allmendinger et al., 2013; Cardozo and Allmendinger, 2013); (C) Photos of transects 1 to 3. (D) Photomicrographs of carbonate host-rock facies: (a) transect 1 coral-rich unit, (b) transect 2 calcarenites, (c) transect 3 calcarenites, and (d) fault rocks 1 and 2 (FR1 and FR2). (E) Pictures of D19 outcrop. (F) Stereographic projection of poles to fractures (density contoured), set 1 faults (orange line) and set 2 faults (red line). (G) Photomicrographs of (a) host rock facies and of $\mathbf{b}$ fault rocks (red stars on the pictures); (H) D19 outcrop including the five faults F1 to F5. 
superposition and overlap principles observed on thin sections using a Technosyn cold cathode luminescence system Model $8200 \mathrm{Mk}$ II coupled to an Olympus BH2 microscope and to a Zeiss MR C5. Micrite micro-fabric and major element composition of two samples from the fault zone, two from the host rock, and one from the D19 karst infilling were measured using Philips XL30 Environmental Scanning Electron Microscope (ESEM) with a beam current set at $20 \mathrm{kV}$ on fresh sample surfaces and on thin sections. To determine stable carbon and oxygen isotopes $\left(\delta^{13} \mathrm{C}\right.$ and $\left.\delta^{18} \mathrm{O}\right)$, 204 microsamples $(<5 \mathrm{mg})$ were drilled, 194 of them were micro-drilled from polished thin sections with an $80 \mu \mathrm{m} \mathrm{di-}$ ameter micro-sampler (Merkantec Micromill) at VU Amsterdam (the Netherlands). We micro-sampled bulk rocks (57), sparitic cements (101), fault rocks (9) and micrite (27). The bulk rock values are related to a nonselective sampling giving information on the whole-rock isotopic values. These values do not capture the signature of isolated cement (Swart, 2015). Carbon and oxygen isotopic values were acquired with Thermo Finnigan Delta + mass spectrometer equipped with a GasBench preparation device at VU Amsterdam. The internationally used standard IAEA-603, with official values of $+2.46 \%$ ofor $\delta^{13} \mathrm{C}$ and $-2.37 \%$ for $\delta^{18} \mathrm{O}$, is measured as a control standard. The standard deviation (SD) of the measurements is, respectively, $<0.1 \%$ and $<0.2 \%$ o for $\delta^{13} \mathrm{C}$ and $\delta^{18} \mathrm{O}$. Ten whole-rock samples were analyzed using a GasBench II connected to a Thermo Fisher Delta V Plus mass spectrometer at the FAU University (Erlangen, Germany). Measurements were calibrated by assigning $\delta^{13} \mathrm{C}$ values of $+1.95 \%$ o to NBS 19 and $-47.3 \%$ o to IAEA-CO9 and $\delta^{18} \mathrm{O}$ values of $-2.20 \%$ o to NBS19. All values are reported in per mil relative to $\mathrm{V}$-PDB.

\section{Results}

\subsection{Microporosity and porosity}

Porosities measured on the 92 samples show a strong decrease towards the fault core (Fig. 3): dropping from more than $10 \%$ in the host carbonates (mean of $15 \%$ and SD of 2.68 for Castellas; mean of $12.3 \%$ and SD of 2.52 for D19) to less than $5 \%$ within fault zones (mean of $4.8 \%$ and SD of 2.07 for Castellas; mean of 3.16\% and SD of 2.35 for D19).

Along transects, some porosity variations occur as follows:

- North of the Castellas Fault, along the $60 \mathrm{~m}$ long transect 2 the porosity is constantly lower than $7 \%$ (mean of $4.4 \%$; SD of 1.53 ; Fig. 3A).

- South of the Castellas Fault, the reduced porosity zone is wider than $40 \mathrm{~m}$ in transect 3 and $30 \mathrm{~m}$ in transect 1 (Fig. 3A). In a $10 \mathrm{~m}$ thick zone from the fault plane, porosity reduction occurs with lower values in transect 1 (average $4.9 \%$ ) than in transect 3 (average $5.6 \%$ ).
- In the D19 Fault zone, the lowest porosity values are found in narrow zones around the faults (less than $2 \mathrm{~m}$ wide) and in the lens between F4 and F5. Though, this porosity decrease is not homogeneous in fault zone, and high values are found north of F1 and F3 (Fig. 3B).

Microscope observation of thin sections impregnated with blue-epoxy resin allowed the identification of a porous rock type with $\phi>10 \%$ mainly in micritized grains as microporosity and moldic porosity (Fig. 3Ca) and a tight rock type with $\phi<5 \%$, where the porosity is mostly linked to barren stylolites (Fig. 3Cb, c).

\subsection{Diagenetic phases}

\subsubsection{Micrite micro-fabric}

Micritized bioclasts, ooids, and peloids were observed after SEM analyzed of two fault zones samples and two host rock samples. Two micro-fabrics of micrite occur with specific crystal shape, sorting, and contacts according to Fournier et al. (2011). Within both fault zones, the micrite is tight, with compact subhedral mosaic crystals less than $10 \mu \mathrm{m}$ wide (MF1; Fig. 4a, b). In the host rock, the micrite is loosely packed and partially coalescent with puntic rarely serrate, subhedral to euhedral crystals less than $5 \mu \mathrm{m}$ wide (MF3; Fig. 4c-e). MF1 correlates with low porosity values $(<5 \%)$, while MF3 with higher porosity (>10\%).

\subsubsection{Diagenetic cements}

Eight different cement stages were identified (Fig. 5). The red stain links to Alizarin Red S coloration and shows that all visible cements are made of calcite, which exhibits variable characteristics (morphology, luminescence, size, and location).

The first two cement phases occur in both fault zones. The first cement ( $\mathrm{C} 0)$ is non-luminescent isopachous calcite of constant thickness $(\sim 10 \mu \mathrm{m})$ around grains (Fig. 5a). The second cement $(\mathrm{C} 1)$ is divided in two sub-phases: a non-luminescent calcite, $\mathrm{C} 1 \mathrm{a}$, with a crystal size ranging from $50 \mu \mathrm{m}$ to more than $200 \mu \mathrm{m}$, a dog-tooth morphology in intergranular spaces, and a bright luminescence calcite, $\mathrm{C} 1 \mathrm{~b}$, covering $\mathrm{C} 1 \mathrm{a}$ with a maximum thickness of $100 \mu \mathrm{m}$ (Fig. 5a, b, d, g). C1b also fills microporosity in micritized grains (Fig. 5b). C1b areal occurrence strongly increases in the Castellas Fault zone.

Five cements or replacive phases extensively occur in the Castellas sector and rarely in the D19 outcrop:

C2 is a sparitic cement, with dull-orange luminescent crystals with a maximum size of $100 \mu \mathrm{m}$ only found in veins of the fault core (Fig. 5b). SEM measurements show the presence of $\mathrm{Si}$ and $\mathrm{Al}$ in the $\mathrm{C} 2$ veins. Most of the Si crystals are automorphic and have black luminescence.

Phantoms of planar-e (euhedral) dolomite crystals (Sibley and Gregg, 1987) with a maximum size of $500 \mu \mathrm{m}$ affect 


\section{A Castellas Fault zone}

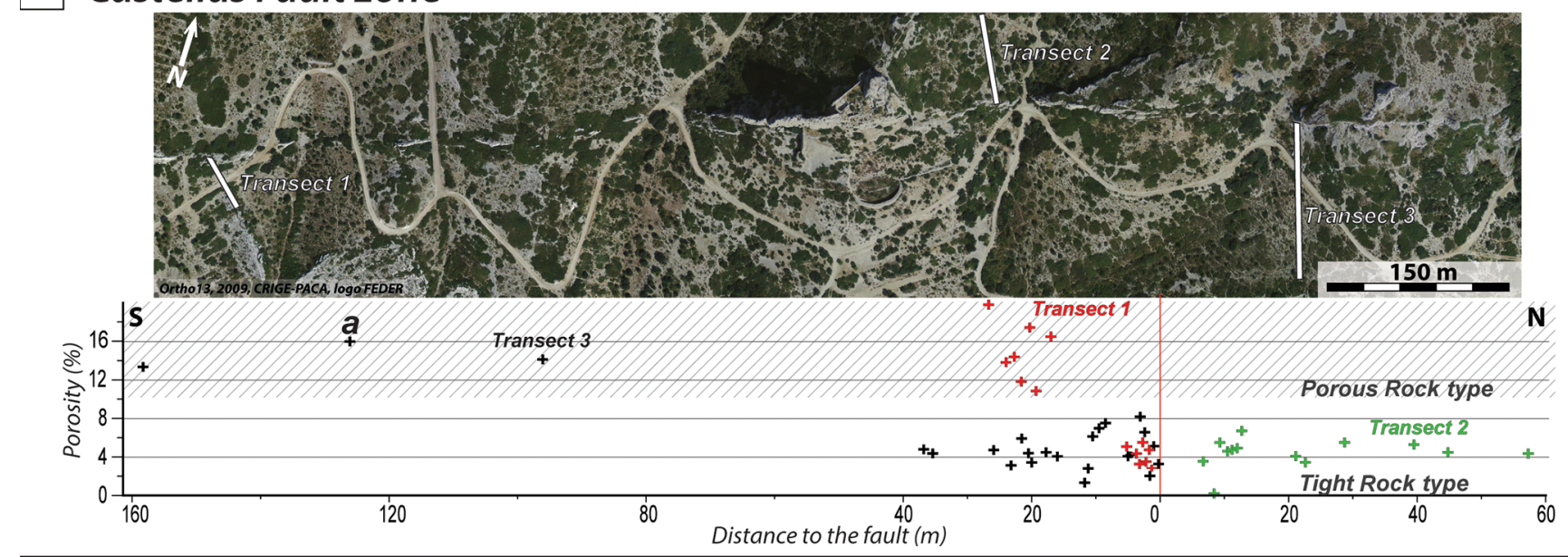

B D19 Fault zone
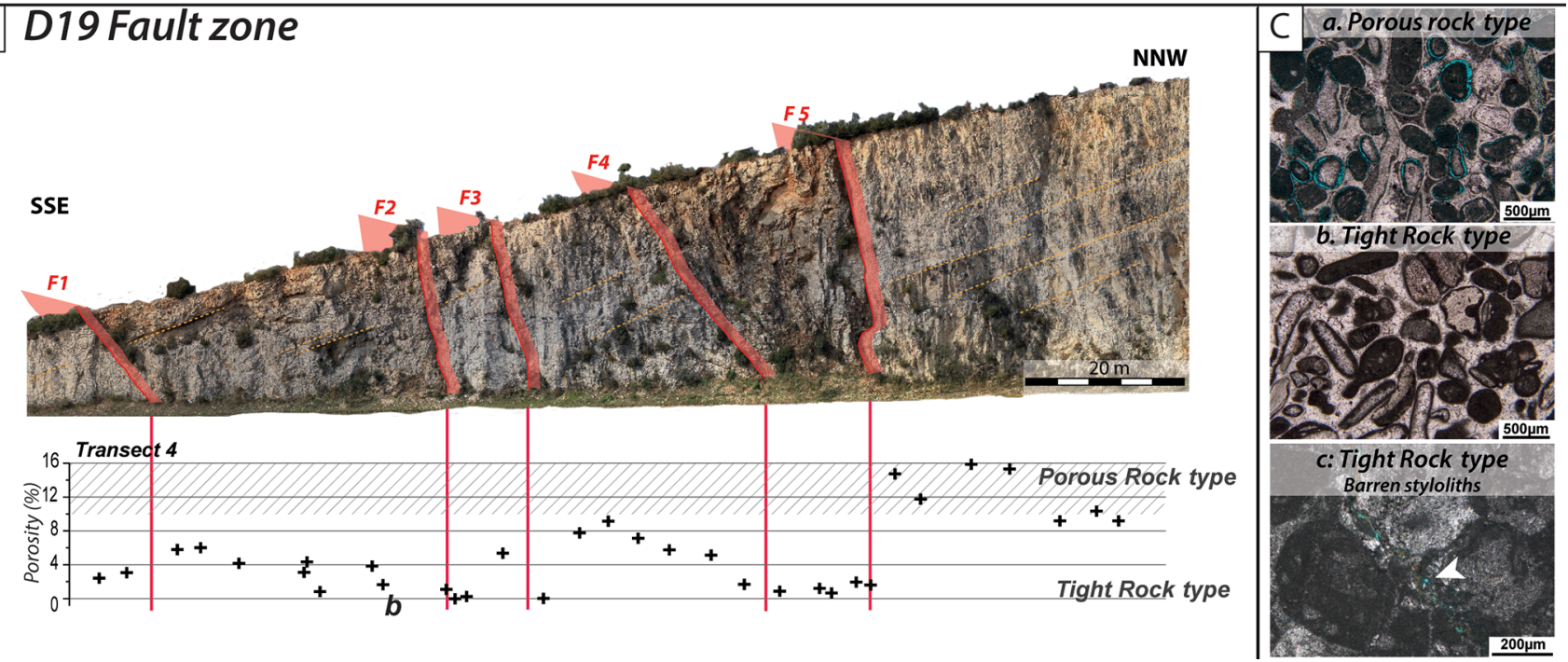

Figure 3. (A) Castellas Fault zone aerial view (Ortho13, 2009, CRIGE-PACA, logo FEDER) and porosity values measured along transect 1 (red cross), transect 2 (green cross), and transect 3 (black cross); (B) porosity values measured along D19 Fault zone; (C) Pore types in the host rock (a) and in the fault zones (b and $\mathbf{c})$.

the matrix of FR1 (Fig. 5e). They are vestiges of a previous dolomitization phase. They have a cloudy appearance caused by solid micritic inclusion inside crystals and can be considered as replacive dolomite (RD; Machel, 2004). Within the FR1 matrix, an important concentration of angular grains of quartz with a maximum size of $300 \mu \mathrm{m}$ is noticed (Fig. 5f).

A blocky calcite C4 (referred to as S2 in Aubert et al., 2019a) is mainly present in veins of the D19 outcrop, in the matrix of FR2, and intergranular and moldic pores (Fig. $5 \mathrm{~g}, \mathrm{~h}$ ). This cement shows zonations of nonluminescent and bright luminescent bands and can be divided in two sparitic sub-phases: $\mathrm{C} 4 \mathrm{a}$ is non-luminescent with some highly luminescent bands, while $\mathrm{C} 4 \mathrm{~b}$ is bright luminescent with some thin non-luminescent zones. C4a occurs in lesser proportion in some veins along transect 2 and 3 of the Castellas Fault.
A sparitic cement $\mathrm{C} 5$, with a red-dull luminescence replaces the RD phase (Fig. 5f).

\subsubsection{Additional diagenetic features}

In addition to cementation phases, other diagenetic elements affected both fault zones. Karst infilling occurs in the F2 fault zone of the D19 outcrop. It is composed of well-sorted grains deposited in laminated layers. This karst deposit presents a stack of alternating micrite-rich and grain-rich layers from the latter composed of former blocky calcite belonging to dissolved grainstones. The laminated layers are affected by veins and stylolites; some of these are deformed due to the grain fall on sediments. Micritic layers have been observed under SEM, and the micrite appears tight with compact subhedral mosaic crystals (Fig. 4f). We observed oxide filling mainly in the Castellas area in dissolution voids affecting 

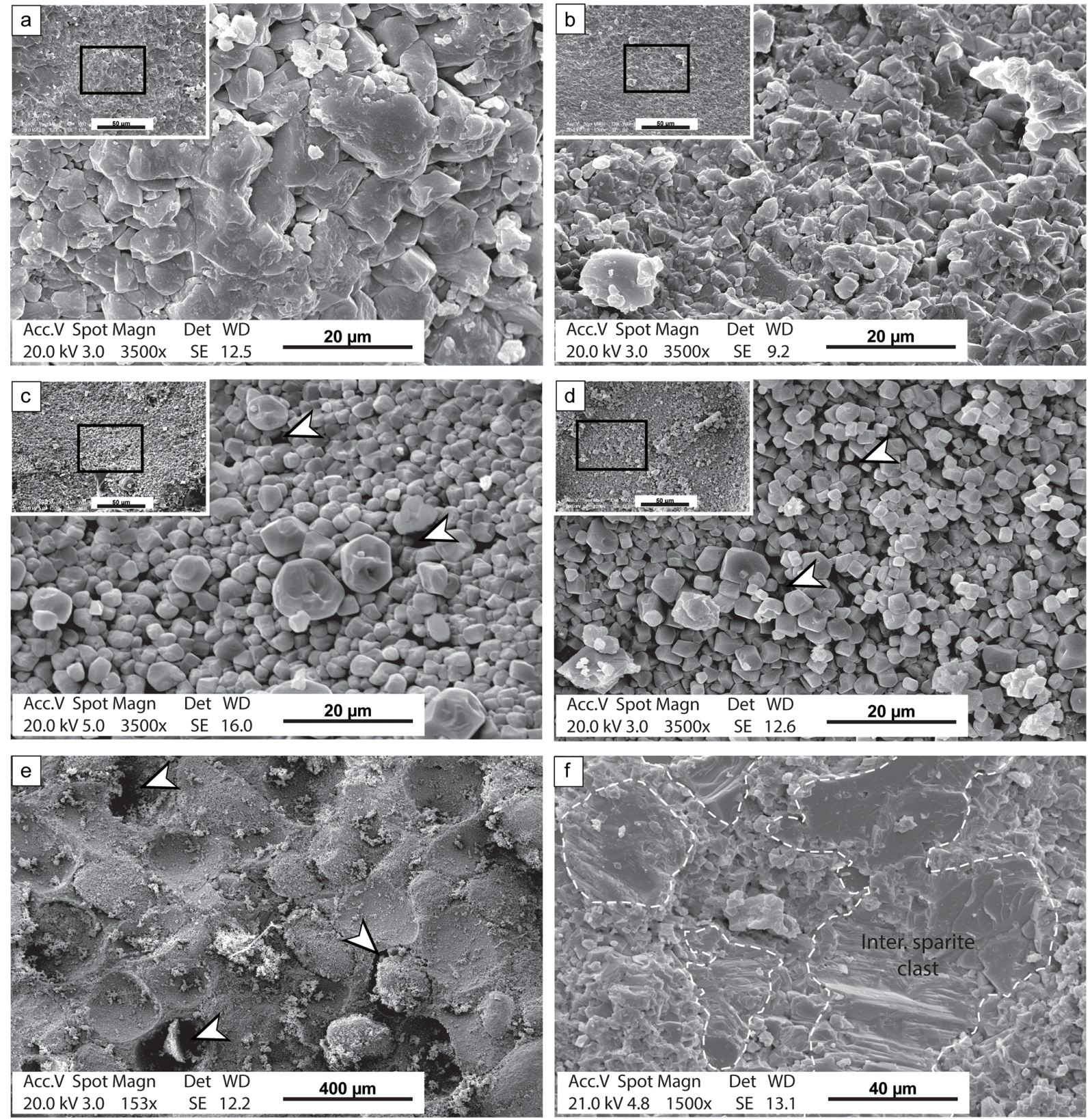

Figure 4. SEM pictures of micrite micro-fabric and microporosity (white arrows); (a) MF1 micrite micro-fabric in Castellas Fault zone (2.5 $\mathrm{m}$ to fault plane); (b) MF1 micrite micro-fabric within D19 Fault zones (2 m away from F5 fault plane); (c) MF3 micrite micro-fabric within Castellas host rock (188 m away from the fault plane); (d) MF3 micrite micro-fabric within D19 host rock (95 m away from F5 fault plane); (e) D19 host rock moldic porosity; (f) karst infilling.

C1a, C1b, and C3 cementation phases and in D19 in karstic fill. The areal amount of oxides increases close to stylolites.

\subsection{Carbon and oxygen isotopes}

Isotope measurements were realized on samples collected along transects of the fault zones. There were 189 measurements of $\mathrm{C}$ and $\mathrm{O}$ isotopes performed on 16 samples and 32 thin sections (Fig. 6a, Table 2).
Sampling was done in bulk rock (66), sparitic cement (101; veins, intergranular volume, and fault rock cements), and fault rocks (10) in order to determine their isotopic signature. Isotopic values range from $-10.40 \%$ to $-3.65 \%$ for $\delta^{18} \mathrm{O}$ and from $-7.20 \%$ to $+1.42 \%$ for $\delta^{13} \mathrm{C}$ (Fig. 6a, b, Table 2). The bulk rock values range from $-9.18 \%$ to $-4.34 \%$ or $\delta^{18} \mathrm{O}$ and from $-4.80 \%$ to $+1.19 \%$ for $\delta^{13} \mathrm{C}$ (Fig. 6a, Table 2). These values are split in two sets. Set 1 includes transects 1 and 3 of the Castellas Fault. Bulk val- 

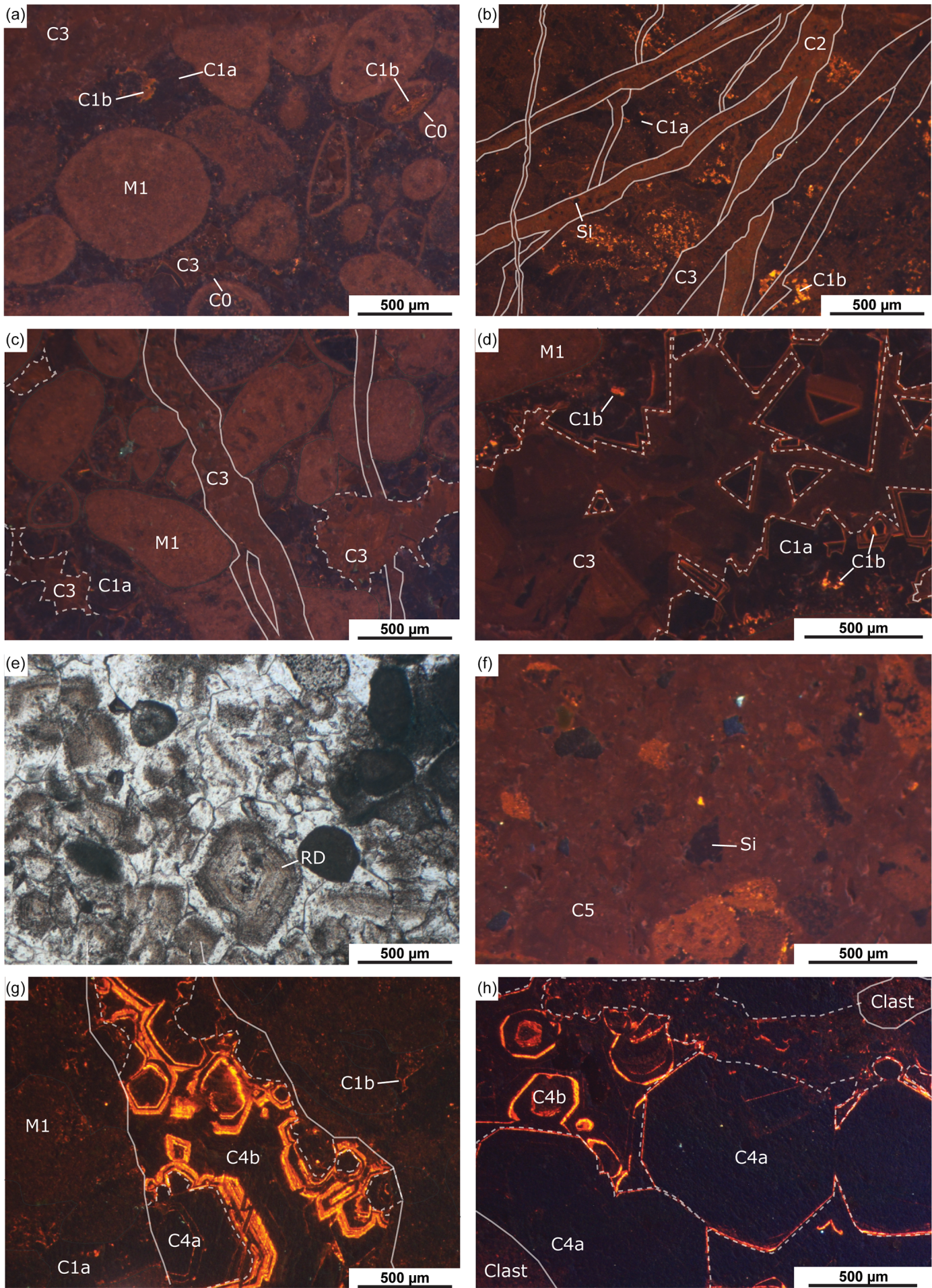

Figure 5. Thin sections under cathodoluminescence. (a) Calcarenite in transect 3 with micritized grain (M1) and intergranular volume cemented with $\mathrm{C} 1 \mathrm{a}$ and b and $\mathrm{C} 3$; (b) $\mathrm{C} 2$ (with $\mathrm{Si}$ ) and $\mathrm{C} 3$ veins affecting Castellas FR1 clasts with micritized grains cemented by $\mathrm{C} 1 \mathrm{~b}$; (c) C3 veins, cements, and intergranular volumes in Castellas Fault zone; (d) C1 (a and b) and C3 cementing moldic porosity of transect 3 calcarenite; (e) FR1 matrix with phantom of cloudy appearance replacive dolomite (RD); (f) FR1 matrix dedolomitized by C5 containing quartz grains; (g) C4 (a and b) cementing vein of D19 Fault zone; (h) matrix of D19 FR2 cemented by C4 (a and b). C3 is a blocky calcite with non- to red-dull luminescence in veins, moldic, and intergranular pores (b-d). This cement also occurs in few veins of D19 sectors but is not restricted only to the fault zone. 
ues range from $-6.07 \%$ to $-4.34 \%$ for $\delta^{18} \mathrm{O}$ and from $-1.41 \%$ to $+1.19 \%$ for $\delta^{13} \mathrm{C}$. Set 2 includes transects 2 (Castellas) and 4 (D19). Bulk values range from $-9.18 \%$ o to $-5.20 \%$ ofor $\delta^{18} \mathrm{O}$ and from $-4.80 \%$ to $-0.60 \%$ for $\delta^{13} \mathrm{C}$ (Fig. 6b, Table 2). In transect 3, the isotopic values only slightly vary, ranging from $-6.13 \%$ o to $-4.50 \%$ or $\delta^{18} \mathrm{O}$ and from $-1.41 \%$ o to $+0.47 \%$ or $\delta^{13} \mathrm{C}$, respectively (Fig. 6c, Table 2). On the contrary, values are more variable along the D19 transect; they range from $-9.18 \%$ o to $-5.20 \%$ for $\delta^{18} \mathrm{O}$ and from $-4.80 \%$ to $-0.60 \%$ o for $\delta^{13} \mathrm{C}$ (Fig. 6c, Table 2). The $\delta^{13} \mathrm{C}$ values are more depleted approaching the faults, especially south of $\mathrm{F} 2$.

Isotopic values of cements filling veins, intergranular volumes, karst infillings, and fault rocks are divided into five groups (Fig. 6a, Table 2).

- Isotopic values of $\mathrm{C} 1$ cement fluctuate from $-6.76 \%$ to $-4.45 \%$ for $\delta^{18} \mathrm{O}$ and from $-1.28 \%$ to $+1.08 \%$ for $\delta^{13} \mathrm{C}$.

- Isotopic values of C3 cement range from $-10.40 \%$ to $-6.73 \%$ of $\delta^{18} \mathrm{O}$ and from $-2.09 \%$ o to $+1.22 \%$ for $\delta^{13} \mathrm{C}$.

- Isotopic values of C4 cement in FR1 and FR2 matrix and in karst infillings range from $-9.18 \%$ o to $-4.60 \%$ o for $\delta^{18} \mathrm{O}$ and from $-5.10 \%$ to $-0.74 \%$ for $\delta^{13} \mathrm{C}$ with a positive covariance between $\delta^{18} \mathrm{O}$ and $\delta^{13} \mathrm{C}$. FR2 matrix values (from $-7.06 \%$ to $-6.55 \%$ o for $\delta^{18} \mathrm{O}$ and from -1.10 to $-2.24 \%$ of $\delta^{13} \mathrm{C}$ ) present slightly less depleted values than karst infillings with mean values of $-7.83 \%$ and $-2.53 \%$ for $\delta^{18} \mathrm{O}$ and $\delta^{13} \mathrm{C}$, respectively (Fig. 6a). In the Castellas Fault, four isotopic values from two veins are enriched with means of $-6.25 \%$ o and $-4.2 \%$ ofor $\delta^{18} \mathrm{O}-0.64 \%$ and $-0.09 \%$ for $\delta^{13} \mathrm{C}$ having similar positive covariance as the other $\mathrm{C} 4$ values.

- Isotopic values of C5 cement, sampled in the FR1 matrix display mean of $-7.49 \%$ for $\delta^{18} \mathrm{O}$ and $-4.01 \%$ for $\delta^{13} \mathrm{C}$ (Fig. 6a).

- Isotopic values of FR3 matrix display a mean of $-5.98 \%$ for $\delta^{18} \mathrm{O}$ and $-6.83 \%$ of $\delta^{13} \mathrm{C}$ (Fig. 6a).

\section{Discussion}

\subsection{Diagenetic evolution of the fault zones}

The chronological relations between cements can be established via crosscutting relations and inclusion principles. Indeed, the veins filled with $\mathrm{C} 2$ cement crosscut $\mathrm{C} 1 \mathrm{a}$ and $\mathrm{C} 1 \mathrm{~b}$ cements (Fig. 5b). Thus, $\mathrm{C} 2$ cementation post-dated $\mathrm{C} 1$ cement. $\mathrm{C} 3$ veins crosscut the $\mathrm{C} 2$ veins but are included within FR1 clasts (Fig. 5b). Hence, C3 cement is prior to FR1 development but is subsequent to $\mathrm{C} 2$ cementation. The fault rock 1
(FR1) is related to the first extensional fault activity, consequently, $\mathrm{C} 1, \mathrm{C} 2$, and $\mathrm{C} 3$ cementation phases occurred prior to the proper fault plane and fault core formation and are related to the fault nucleation. Replacive dolomite is found within the FR1 matrix (Fig. 5e); therefore, it developed after FR1 formation. Finally, the $\mathrm{C} 4$ cement can be noticed within the FR2 matrix, indicating that $\mathrm{C} 4$ cementation event post-dated FR2 formation. The fault rock 2 (FR2) developed during strike-slip reactivation of the studied faults. The combined superposition, overlap, crosscutting principles, and isotopic signature of cements brought out the chronology between phases and revealed the paragenetic sequence (Fig. 7).

The Urgonian carbonates in La Fare anticline underwent three major diagenetic events, which impacted the host rock and/or the fault zones. We discriminate among diagenetic events that occurred before and during faulting.

\subsubsection{Pre-fault diagenesis - microporosity development}

During the Upper Barremian, just after deposition, microbore organisms at the sediment-water interface enhanced the formation of micritic calcitic envelopes on bioclasts, ooids, and peloids (Purser, 1980; Reid and Macintyre, 2000; Samankassou et al., 2005; Vincent et al., 2007). This micritization in marine conditions is typical for the Urgonian lowenergy inner-platform environment (Fournier et al., 2011; Masse, 1976). Subsequently, C0 cement formed around grains giving rise to a solid envelop inducing the preservation of the original grain shape during the later burial compaction (Step 0 in Fig. 8). However, the majority of isotopic values do not fit in the Barremian seawater calcite box, which ranges from $-4.00 \%$ to $-1.00 \%$ for $\delta^{18} \mathrm{O}$ and from $+1.00 \%$ to $+3.00 \%$ or for $\delta^{13} \mathrm{C}$ (Fouke et al., 1996; Godet et al., 2006). Only two data points pertaining to micritized grains show isotopic values close the Barremian seawater calcite. The isotopic depletion of other data indicates the slight impact of $\mathrm{C} 0$ cementation on isotopic values.

The next sub-phase of cementation C1a partly fills intergranular porosity. This non-luminescent cement with isotopic values ranging from $-6.8 \%$ to $-3.9 \%$ for $\delta^{18} \mathrm{O}$ and from $-1.0 \%$ to $+1.3 \%$ for $\delta^{13} \mathrm{C}$ is characteristic of mixed fluids. Léonide et al. (2014) measured a calcite cement S1 near La Fare anticline with similar luminescence and isotopic range values (mean: $\delta^{18} \mathrm{O}=-5.49 \% ; \delta^{13} \mathrm{C}=+2.34 \%$ ). These authors linked this cementation phase to a shallowburial meteoric fluid circulation under equatorial climate during Durancian uplift. This diagenetic event led to micrite recrystallization and to the development of microporosity (MF3). Since La Fare carbonates were exhumed at that time (Léonide et al., 2014), the meteoric fluids led to similar diagenetic modifications (Step 1 in Fig. 8):

i. micrite recrystallization and microporosity MF3 setup by Ostwald ripening processes (Fig. 9B1a; Ostwald, 1886; Volery et al., 2010); 
Table 2. Carbon and oxygen isotope values of bulk carbonates for Castellas Fault zone and D19 Fault zones. B: bulk measurement; M: micrite value; $\mathrm{C} 1, \mathrm{C} 3, \mathrm{C} 4$, and $\mathrm{C} 5$ : cement isotopic value; $\mathrm{FR}$ : fault rock isotopic value.

\begin{tabular}{|c|c|c|c|c|c|}
\hline Transect & Sample & $\begin{array}{r}\delta^{13} \mathrm{C} \\
(\% \circ \mathrm{VPDB})\end{array}$ & $\begin{array}{r}\delta^{18} \mathrm{O} \\
(\% \circ \mathrm{VPDB})\end{array}$ & Class & $\begin{array}{l}\text { Distance } \\
\text { to F. (m) }\end{array}$ \\
\hline Transect 1 (Cast.) & 201 & 1.19 & -4.34 & B & 1.3 \\
\hline Transect 1 (Cast.) & 201 & 1.02 & -6.62 & $\mathrm{C} 1$ & 1.3 \\
\hline Transect 1 (Cast.) & 201 & 1.31 & -3.94 & $\mathrm{M}$ & 1.3 \\
\hline Transect 1 (Cast.) & 201 & 1.37 & -3.65 & M & 1.3 \\
\hline Transect 1 (Cast.) & 213 & -0.68 & -5.24 & $\mathrm{~B}$ & 22.7 \\
\hline Transect 1 (Cast.) & 213 & -0.58 & -5.10 & $\mathrm{~B}$ & 22.7 \\
\hline Transect 1 (Cast.) & 213 & -0.18 & -6.09 & $\mathrm{C} 1$ & 22.7 \\
\hline Transect 1 (Cast.) & 213 & 0.03 & -4.45 & $\mathrm{C} 1$ & 22.7 \\
\hline Transect 1 (Cast.) & 213 & 0.09 & -4.77 & $\mathrm{C} 1$ & 22.7 \\
\hline Transect 1 (Cast.) & 213 & -2.09 & -6.92 & $\mathrm{C} 4$ & 22.7 \\
\hline Transect 1 (Cast.) & 213 & -0.68 & -4.92 & $\mathrm{M}$ & 22.7 \\
\hline Transect 2 (Cast.) & c3b17 & -0.52 & -5.95 & $\mathrm{~B}$ & 4.6 \\
\hline Transect 2 (Cast.) & c3b17 & -2.07 & -6.38 & $\mathrm{C} 4$ & 4.6 \\
\hline Transect 2 (Cast.) & $\mathrm{c} 3 \mathrm{~b} 7$ & -0.64 & -5.51 & $\mathrm{~B}$ & 9.3 \\
\hline Transect 2 (Cast.) & $\mathrm{c} 3 \mathrm{~b} 26$ & -3.76 & -6.26 & $\mathrm{~B}$ & 22.6 \\
\hline Transect 2 (Cast.) & $\mathrm{c} 3 \mathrm{~b} 26$ & -2.85 & -5.58 & $\mathrm{C} 4$ & 22.6 \\
\hline Transect 2 (Cast.) & $\mathrm{c} 3 \mathrm{~b} 26$ & -1.31 & -4.69 & $\mathrm{~B}$ & 57.3 \\
\hline Transect 2 (Cast.) & $\mathrm{c} 3 \mathrm{~b} 7$ & -1.76 & -6.31 & $\mathrm{C} 1$ & 57.3 \\
\hline Transect 2 (Cast.) & $\mathrm{c} 3 \mathrm{~b} 7$ & -1.28 & -6.46 & $\mathrm{C} 1$ & 57.3 \\
\hline Transect 2 (Cast.) & $\mathrm{c} 3 \mathrm{~b} 26$ & -2.35 & -5.22 & $\mathrm{M}$ & 57.3 \\
\hline Transect 2 (Cast.) & $\mathrm{c} 3 \mathrm{~b} 26$ & -1.70 & -4.75 & M & 57.3 \\
\hline Transect 3 (Cast.) & 327 & -0.24 & -7.55 & $\mathrm{C} 3$ & 0.3 \\
\hline Transect 3 (Cast.) & 325 & -1.90 & -9.06 & $\mathrm{C} 3$ & 0.3 \\
\hline Transect 3 (Cast.) & 325 & -1.69 & -8.95 & $\mathrm{C} 3$ & 0.3 \\
\hline Transect 3 (Cast.) & 327 & -3.11 & -8.09 & $\mathrm{C} 4$ & 0.3 \\
\hline Transect 3 (Cast.) & 327 & 0.47 & -5.40 & $\mathrm{~B}$ & 1.0 \\
\hline Transect 3 (Cast.) & 327 & -0.18 & -7.95 & $\mathrm{C} 3$ & 1.0 \\
\hline Transect 3 (Cast.) & 327 & -0.17 & -7.41 & $\mathrm{C} 3$ & 1.0 \\
\hline Transect 3 (Cast.) & 328 & 0.10 & -5.74 & $\mathrm{C} 1$ & 1.6 \\
\hline Transect 3 (Cast.) & 328 & -1.32 & -8.18 & $\mathrm{C} 3$ & 1.6 \\
\hline Transect 3 (Cast.) & 328 & -0.59 & -7.77 & $\mathrm{C} 3$ & 1.6 \\
\hline Transect 3 (Cast.) & 328 & -0.42 & -7.74 & $\mathrm{C} 3$ & 1.6 \\
\hline Transect 3 (Cast.) & 328 & -0.13 & -9.26 & $\mathrm{C} 3$ & 1.6 \\
\hline Transect 3 (Cast.) & 328 & 0.02 & -8.83 & $\mathrm{C} 3$ & 1.6 \\
\hline Transect 3 (Cast.) & 328 & 0.29 & -8.70 & $\mathrm{C} 3$ & 1.6 \\
\hline Transect 3 (Cast.) & 328 & 0.42 & -8.73 & $\mathrm{C} 3$ & 1.6 \\
\hline Transect 3 (Cast.) & 328 & 0.50 & -7.89 & $\mathrm{C} 3$ & 1.6 \\
\hline Transect 3 (Cast.) & 328 & 1.22 & -8.18 & $\mathrm{C} 3$ & 1.6 \\
\hline Transect 3 (Cast.) & 333 & -1.84 & -8.67 & $\mathrm{C} 3$ & 1.6 \\
\hline Transect 3 (Cast.) & 333 & -0.96 & -7.89 & $\mathrm{C} 3$ & 1.6 \\
\hline Transect 3 (Cast.) & 328 & -0.14 & -4.17 & $\mathrm{C} 4$ & 1.6 \\
\hline Transect 3 (Cast.) & 328 & -0.05 & -4.23 & $\mathrm{C} 4$ & 1.6 \\
\hline Transect 3 (Cast.) & 329 & 0.16 & -4.95 & $\mathrm{~B}$ & 2.4 \\
\hline Transect 3 (Cast.) & 333 & -0.25 & -6.38 & $\mathrm{C} 1$ & 4.6 \\
\hline Transect 3 (Cast.) & 333 & -0.12 & -6.17 & $\mathrm{C} 1$ & 4.6 \\
\hline Transect 3 (Cast.) & 333 & -0.62 & -8.52 & $\mathrm{C} 3$ & 4.6 \\
\hline Transect 3 (Cast.) & 333 & -0.12 & -5.67 & M & 4.6 \\
\hline Transect 3 (Cast.) & 333 & -0.02 & -4.48 & M & 4.6 \\
\hline Transect 3 (Cast.) & 333 & 0.42 & -4.60 & M & 4.6 \\
\hline Transect 3 (Cast.) & 337 & 0.19 & -5.59 & $\mathrm{~B}$ & 9.5 \\
\hline Transect 3 (Cast.) & 302 & -0.53 & -4.50 & $\mathrm{~B}$ & 11.8 \\
\hline Transect 3 (Cast.) & 302 & -0.49 & -4.74 & $\mathrm{~B}$ & 11.8 \\
\hline Transect 3 (Cast.) & 302 & -0.62 & -10.38 & $\mathrm{C} 3$ & 11.8 \\
\hline
\end{tabular}


Table 2. Continued.

\begin{tabular}{|c|c|c|c|c|c|}
\hline Transect & Sample & $\begin{array}{r}\delta^{13} \mathrm{C} \\
(\% \circ \mathrm{VPDB})\end{array}$ & $\begin{array}{r}\delta^{18} \mathrm{O} \\
(\% \circ \mathrm{VPDB})\end{array}$ & Class & $\begin{array}{l}\text { Distance } \\
\text { to F. (m) }\end{array}$ \\
\hline Transect 3 (Cast.) & 302 & -0.49 & -10.02 & $\mathrm{C} 3$ & 11.8 \\
\hline Transect 3 (Cast.) & 305 & 0.33 & -4.38 & $\mathrm{~B}$ & 16.0 \\
\hline Transect 3 (Cast.) & 306 & 0.21 & -4.35 & B & 17.8 \\
\hline Transect 3 (Cast.) & 307 & -0.01 & -4.46 & B & 18.2 \\
\hline Transect 3 (Cast.) & 308 & -0.57 & -4.95 & B & 20.0 \\
\hline Transect 3 (Cast.) & 308 & -1.44 & -9.11 & $\mathrm{C} 3$ & 20.0 \\
\hline Transect 3 (Cast.) & 308 & -0.23 & -10.40 & $\mathrm{C} 3$ & 20.0 \\
\hline Transect 3 (Cast.) & 308 & -0.22 & -10.08 & $\mathrm{C} 3$ & 20.0 \\
\hline Transect 3 (Cast.) & 309 & -1.41 & -4.87 & $\mathrm{~B}$ & 20.5 \\
\hline Transect 3 (Cast.) & 309 & -0.52 & -5.01 & B & 20.5 \\
\hline Transect 3 (Cast.) & 309 & -0.15 & -4.82 & $\mathrm{C} 1$ & 20.5 \\
\hline Transect 3 (Cast.) & 309 & -1.56 & -7.96 & $\mathrm{C} 3$ & 20.5 \\
\hline Transect 3 (Cast.) & 309 & -1.55 & -8.01 & $\mathrm{C} 3$ & 20.5 \\
\hline Transect 3 (Cast.) & 312 & 0.12 & -4.81 & $\mathrm{~B}$ & 23.2 \\
\hline Transect 3 (Cast.) & 314 & -0.71 & -5.30 & $\mathrm{~B}$ & 25.9 \\
\hline Transect 3 (Cast.) & 314 & -0.80 & -10.09 & $\mathrm{C} 3$ & 25.9 \\
\hline Transect 3 (Cast.) & 314 & -0.49 & -9.90 & $\mathrm{C} 3$ & 25.9 \\
\hline Transect 3 (Cast.) & 314 & -0.47 & -10.29 & $\mathrm{C} 3$ & 25.9 \\
\hline Transect 3 (Cast.) & 314 & -0.40 & -9.97 & $\mathrm{C} 3$ & 25.9 \\
\hline Transect 3 (Cast.) & 314 & 0.06 & -10.30 & $\mathrm{C} 3$ & 25.9 \\
\hline Transect 3 (Cast.) & 316 & -1.24 & -5.50 & $\mathrm{~B}$ & 29.2 \\
\hline Transect 3 (Cast.) & 316 & -1.00 & -5.48 & $\mathrm{~B}$ & 29.2 \\
\hline Transect 3 (Cast.) & 316 & -0.22 & -4.79 & $\mathrm{~B}$ & 29.2 \\
\hline Transect 3 (Cast.) & 316 & -1.02 & -10.21 & $\mathrm{C} 3$ & 29.2 \\
\hline Transect 3 (Cast.) & 316 & -0.18 & -9.31 & $\mathrm{C} 3$ & 29.2 \\
\hline Transect 3 (Cast.) & 316 & 0.30 & -10.37 & $\mathrm{C} 3$ & 29.2 \\
\hline Transect 3 (Cast.) & 318 & -0.28 & -4.53 & $\mathrm{~B}$ & 35.4 \\
\hline Transect 3 (Cast.) & 320 & -0.68 & -5.79 & B & 96.1 \\
\hline Transect 3 (Cast.) & 322 & -0.88 & -6.07 & $\mathrm{~B}$ & 158.0 \\
\hline Transect 3 (Cast.) & 323 & -0.65 & -5.37 & B & 188.0 \\
\hline Castellas (ZF1) & $\mathrm{Z} 1.1$ & 0.17 & -5.26 & $\mathrm{C} 1$ & 0.0 \\
\hline Castellas (ZF1) & $\mathrm{Z} 1.1$ & 0.39 & -5.23 & $\mathrm{C} 1$ & 0.0 \\
\hline Castellas (ZF1) & $\mathrm{Z} 1.1$ & 0.46 & -4.70 & $\mathrm{C} 1$ & 0.0 \\
\hline Castellas (ZF1) & $\mathrm{Z} 1.2$ & 0.21 & -5.98 & $\mathrm{C} 1$ & 0.0 \\
\hline Castellas (ZF1) & $\mathrm{Z} 1.1$ & -0.55 & -6.40 & $\mathrm{C} 4$ & 0.0 \\
\hline Castellas (ZF1) & $\mathrm{Z} 1.1$ & -0.52 & -6.10 & $\mathrm{C} 4$ & 0.0 \\
\hline Castellas (ZF1) & $\mathrm{Z} 1.2$ & -4.12 & -7.45 & $\mathrm{C} 5$ & 0.0 \\
\hline Castellas (ZF1) & $\mathrm{Z} 1.2$ & -0.15 & -4.99 & FR & 0.0 \\
\hline Castellas (ZF1) & $\mathrm{Z} 1.2$ & 0.39 & -4.73 & M & 0.0 \\
\hline Castellas (ZF1) & $\mathrm{Z} 1.2$ & 0.61 & -5.77 & $\mathrm{M}$ & 0.0 \\
\hline Castellas (ZF1) & $\mathrm{Z} 1.1$ & 0.78 & -6.16 & M & 0.0 \\
\hline Castellas (ZF2) & $\mathrm{Z} 2.2$ & 0.77 & -5.38 & $\mathrm{C} 1$ & 0.0 \\
\hline Castellas (ZF2) & $\mathrm{Z} 2.7$ & -1.40 & -9.52 & $\mathrm{C} 3$ & 0.0 \\
\hline Castellas (ZF2) & $\mathrm{Z} 2.7$ & -4.38 & -7.15 & $\mathrm{C} 5$ & 0.0 \\
\hline Castellas (ZF2) & $\mathrm{Z} 2.7$ & -3.97 & -7.13 & $\mathrm{C} 5$ & 0.0 \\
\hline Castellas (ZF2) & $\mathrm{Z} 2.7$ & -3.78 & -8.04 & $\mathrm{C} 5$ & 0.0 \\
\hline Castellas (ZF2) & $\mathrm{Z} 2.7$ & -3.56 & -7.86 & $\mathrm{C} 5$ & 0.0 \\
\hline Castellas (ZF2) & $\mathrm{Z} 2.7$ & -3.24 & -7.48 & $\mathrm{C} 5$ & 0.0 \\
\hline Castellas (ZF2) & $\mathrm{Z} 2.7$ & -3.23 & -8.54 & $\mathrm{C} 5$ & 0.0 \\
\hline Castellas (ZF2) & $\mathrm{Z} 2.2$ & 0.58 & -5.47 & FR & 0.0 \\
\hline Castellas (ZF2) & $\mathrm{Z} 2.2$ & 0.92 & -4.91 & FR & 0.0 \\
\hline Castellas (ZF2) & $\mathrm{Z} 2.7$ & -1.68 & -5.63 & FR & 0.0 \\
\hline Castellas (ZF2) & $\mathrm{Z} 2.7$ & -2.24 & -6.55 & FR & 0.0 \\
\hline
\end{tabular}


Table 2. Continued.

\begin{tabular}{|c|c|c|c|c|c|}
\hline Transect & Sample & $\begin{array}{r}\delta^{13} \mathrm{C} \\
(\% \circ \mathrm{VPDB})\end{array}$ & $\begin{array}{r}\delta^{18} \mathrm{O} \\
(\% \circ \mathrm{VPDB})\end{array}$ & Class & $\begin{array}{l}\text { Distance } \\
\text { to F. (m) }\end{array}$ \\
\hline Castellas (ZF2) & $\mathrm{Z} 2.7$ & -3.18 & -7.38 & $\mathrm{M}$ & 0.0 \\
\hline Castellas (ZF2) & $\mathrm{Z} 2.7$ & -2.86 & -6.03 & FR & 1.0 \\
\hline Castellas (ZF5) & Z5.4 & 0.27 & -8.25 & $\mathrm{C} 3$ & 0.0 \\
\hline Castellas (ZF5) & Z5.4 & 0.31 & -7.87 & $\mathrm{C} 3$ & 0.0 \\
\hline Castellas (ZF5) & Z5.4 & 0.32 & -8.23 & $\mathrm{C} 3$ & 0.0 \\
\hline Castellas (ZF5) & $\mathrm{Z} 5.4$ & 1.06 & -6.34 & $\mathrm{C} 1$ & 0.4 \\
\hline Castellas (ZF5) & Z5.4 & 1.08 & -6.76 & $\mathrm{C} 1$ & 0.4 \\
\hline Castellas (ZF5) & $\mathrm{Z} 5.4$ & 1.05 & -7.13 & FR & 0.4 \\
\hline Castellas (ZF5) & Z5.4 & 1.37 & -6.03 & FR & 0.4 \\
\hline Castellas (ZF5) & Z5.4 & 1.42 & -6.15 & FR & 0.4 \\
\hline Transect & Sample & $\begin{array}{r}\delta^{13} \mathrm{C} \\
(\% \circ \mathrm{VPDB})\end{array}$ & $\begin{array}{r}\delta^{18} \mathrm{O} \\
(\% \circ \mathrm{VPDB})\end{array}$ & Class & $\begin{array}{l}\text { Distance } \\
\text { to F1 (m) }\end{array}$ \\
\hline Transect 4 (D19) & $3 \mathrm{~B}$ & -0.81 & -6.52 & B & 0.0 \\
\hline Transect 4 (D19) & $3 \mathrm{~B}$ & -1.20 & -6.50 & $\mathrm{C} 1$ & 0.0 \\
\hline Transect 4 (D19) & 3B & -1.02 & -6.33 & $\mathrm{C} 1$ & 0.0 \\
\hline Transect 4 (D19) & $3 \mathrm{~B}$ & 0.11 & -6.25 & $\mathrm{C} 1$ & 0.0 \\
\hline Transect 4 (D19) & $3 \mathrm{~B}$ & -0.74 & -6.23 & M & 0.0 \\
\hline Transect 4 (D19) & 9 & -2.32 & -7.30 & $\mathrm{~B}$ & 9.2 \\
\hline Transect 4 (D19) & $13 \mathrm{a}$ & -3.44 & -8.11 & $\mathrm{~B}$ & 14.3 \\
\hline Transect 4 (D19) & $13 \mathrm{a}$ & -2.96 & -7.93 & $\mathrm{~B}$ & 14.3 \\
\hline Transect 4 (D19) & $13 \mathrm{C}$ & -2.97 & -7.62 & $\mathrm{M}$ & 14.3 \\
\hline Transect 4 (D19) & $13 \mathrm{C}$ & -2.86 & -7.79 & $\mathrm{M}$ & 14.3 \\
\hline Transect 4 (D19) & $13 \mathrm{C}$ & -2.70 & -8.12 & M & 14.3 \\
\hline Transect 4 (D19) & $13 \mathrm{C}$ & -2.67 & -7.96 & $\mathrm{M}$ & 14.3 \\
\hline Transect 4 (D19) & $13 \mathrm{C}$ & -2.66 & -8.16 & $\mathrm{M}$ & 14.3 \\
\hline Transect 4 (D19) & $13 \mathrm{C}$ & -2.50 & -7.77 & $\mathrm{M}$ & 14.3 \\
\hline Transect 4 (D19) & $13 \mathrm{C}$ & -1.54 & -8.98 & $\mathrm{M}$ & 14.3 \\
\hline Transect 4 (D19) & 17 & -2.58 & -7.68 & $\mathrm{~B}$ & 18.7 \\
\hline Transect 4 (D19) & $14 \mathrm{~A}$ & -1.97 & -6.38 & $\mathrm{~B}$ & 18.7 \\
\hline Transect 4 (D19) & $14 \mathrm{~A}$ & -1.87 & -6.74 & $\mathrm{~B}$ & 18.7 \\
\hline Transect 4 (D19) & $15 \mathrm{~B}$ & -2.23 & -7.43 & $\mathrm{~B}$ & 18.7 \\
\hline Transect 4 (D19) & 17 & -1.05 & -6.40 & $\mathrm{C} 1$ & 18.7 \\
\hline Transect 4 (D19) & $14 \mathrm{~A}$ & -1.77 & -6.74 & $\mathrm{C} 1$ & 18.7 \\
\hline Transect 4 (D19) & $14 \mathrm{~A}$ & -2.42 & -6.43 & $\mathrm{C} 4$ & 18.7 \\
\hline Transect 4 (D19) & $14 \mathrm{~A}$ & -2.06 & -6.67 & $\mathrm{C} 4$ & 18.7 \\
\hline Transect 4 (D19) & 21 & -2.23 & -6.54 & $\mathrm{~B}$ & 24.4 \\
\hline Transect 4 (D19) & RSG & -1.90 & -7.66 & $\mathrm{~B}$ & 28.4 \\
\hline Transect 4 (D19) & RSG & -1.70 & -7.83 & $\mathrm{~B}$ & 28.4 \\
\hline Transect 4 (D19) & RSD & -2.87 & -7.10 & B & 29.5 \\
\hline Transect 4 (D19) & RSD & -2.76 & -7.14 & $\mathrm{~B}$ & 29.5 \\
\hline Transect 4 (D19) & RSD & -0.93 & -9.40 & $\mathrm{C} 3$ & 29.5 \\
\hline Transect 4 (D19) & RSF1 & -2.40 & -7.28 & $\mathrm{~B}$ & 34.7 \\
\hline Transect 4 (D19) & RSF2 & -2.14 & -7.39 & $\mathrm{~B}$ & 34.7 \\
\hline Transect 4 (D19) & RSF2 & -1.78 & -7.27 & $\mathrm{~B}$ & 34.7 \\
\hline Transect 4 (D19) & RSF1 & -1.03 & -9.44 & $\mathrm{C} 3$ & 34.7 \\
\hline Transect 4 (D19) & RSF2 & -1.93 & -8.05 & $\mathrm{C} 3$ & 34.7 \\
\hline Transect 4 (D19) & RSF2 & -0.59 & -9.40 & $\mathrm{C} 3$ & 34.7 \\
\hline Transect 4 (D19) & RSF2 & -2.95 & -8.14 & $\mathrm{C} 4$ & 34.7 \\
\hline Transect 4 (D19) & RSE 1 & -2.53 & -7.33 & $\mathrm{~B}$ & 35.0 \\
\hline Transect 4 (D19) & RSE 2 & -2.59 & -7.41 & $\mathrm{~B}$ & 35.0 \\
\hline Transect 4 (D19) & RSE 1 & -1.71 & -7.68 & $\mathrm{C} 3$ & 35.0 \\
\hline
\end{tabular}


Table 2. Continued.

\begin{tabular}{|c|c|c|c|c|c|}
\hline Transect & Sample & $\begin{array}{r}\delta^{13} \mathrm{C} \\
(\% \circ \mathrm{VPDB})\end{array}$ & $\begin{array}{r}\delta^{18} \mathrm{O} \\
(\% \circ \mathrm{VPDB})\end{array}$ & Class & $\begin{array}{l}\text { Distance } \\
\text { to F1 (m) }\end{array}$ \\
\hline Transect 4 (D19) & RSE 2 & -1.84 & -6.73 & $\mathrm{C} 3$ & 35.0 \\
\hline Transect 4 (D19) & 57 & -2.07 & -5.93 & $\mathrm{~B}$ & 38.1 \\
\hline Transect 4 (D19) & 57 & -1.94 & -5.87 & $\mathrm{~B}$ & 38.1 \\
\hline Transect 4 (D19) & 57 & -1.83 & -7.06 & $\mathrm{C} 3$ & 38.1 \\
\hline Transect 4 (D19) & 57 & -1.10 & -6.75 & $\mathrm{C} 3$ & 38.1 \\
\hline Transect 4 (D19) & 57 & -4.02 & -7.04 & $\mathrm{C} 4$ & 38.1 \\
\hline Transect 4 (D19) & 57 & -2.17 & -5.72 & $\mathrm{C} 4$ & 38.1 \\
\hline Transect 4 (D19) & 57 & -1.58 & -6.52 & FR & 38.1 \\
\hline Transect 4 (D19) & 57 & -7.20 & -5.68 & M & 38.1 \\
\hline Transect 4 (D19) & 57 & -7.13 & -5.90 & M & 38.1 \\
\hline Transect 4 (D19) & $28 b$ & -1.03 & -7.21 & $\mathrm{~B}$ & 39.3 \\
\hline Transect 4 (D19) & $28 b$ & -1.03 & -6.10 & $\mathrm{C} 3$ & 39.3 \\
\hline Transect 4 (D19) & $28 b$ & -4.09 & -6.92 & $\mathrm{C} 4$ & 39.3 \\
\hline Transect 4 (D19) & $28 b$ & -2.58 & -7.40 & $\mathrm{C} 4$ & 39.3 \\
\hline Transect 4 (D19) & $28 b$ & -2.47 & -7.54 & $\mathrm{C} 4$ & 39.3 \\
\hline Transect 4 (D19) & $30 \mathrm{a}$ & -1.61 & -7.04 & $\mathrm{~B}$ & 42.6 \\
\hline Transect 4 (D19) & $30 \mathrm{a}$ & -1.41 & -6.87 & $\mathrm{~B}$ & 42.6 \\
\hline Transect 4 (D19) & $30 \mathrm{a}$ & -3.23 & -7.03 & $\mathrm{C} 4$ & 42.6 \\
\hline Transect 4 (D19) & $30 \mathrm{a}$ & -2.89 & -7.45 & $\mathrm{C} 4$ & 42.6 \\
\hline Transect 4 (D19) & $24 a$ & -1.21 & -7.52 & $\mathrm{~B}$ & 51.1 \\
\hline Transect 4 (D19) & $27 b$ & -1.92 & -7.48 & B & 57.9 \\
\hline Transect 4 (D19) & 31 & -1.24 & -6.44 & $\mathrm{~B}$ & 65.0 \\
\hline Transect 4 (D19) & 32 & -1.75 & -7.50 & $\mathrm{~B}$ & 67.4 \\
\hline Transect 4 (D19) & 34 & -1.79 & -7.49 & $\mathrm{~B}$ & 72.2 \\
\hline Transect 4 (D19) & 36 & -1.32 & -7.21 & $\mathrm{~B}$ & 77.8 \\
\hline Transect 4 (D19) & 38 & -1.73 & -7.59 & $\mathrm{~B}$ & 81.5 \\
\hline Transect 4 (D19) & 62 & -1.96 & -7.56 & $\mathrm{~B}$ & 86.0 \\
\hline Transect 4 (D19) & 42 & -0.81 & -6.80 & $\mathrm{~B}$ & 91.9 \\
\hline Transect 4 (D19) & 63 & -0.55 & -5.50 & $\mathrm{~B}$ & 124.0 \\
\hline Transect 4 (D19) & 64 & -1.17 & -5.88 & $\mathrm{~B}$ & 160.0 \\
\hline Transect 4 (D19) & 65 & -1.10 & -6.57 & B & 197.0 \\
\hline Transect 4 (D19) & 66 & -1.31 & -5.21 & $\mathrm{~B}$ & 236.0 \\
\hline Transect 4 (D19) & $60 a$ & -3.06 & -9.18 & $\mathrm{~B}$ & 255.2 \\
\hline Transect 4 (D19) & $60 \mathrm{~B}$ & -4.80 & -8.47 & $\mathrm{~B}$ & 255.2 \\
\hline Transect 4 (D19) & $60 \mathrm{~B}$ & -4.66 & -8.92 & $\mathrm{~B}$ & 255.2 \\
\hline Transect 4 (D19) & 61 & -1.53 & -9.87 & $\mathrm{C} 3$ & 255.2 \\
\hline Transect 4 (D19) & 61 & -1.36 & -9.89 & $\mathrm{C} 3$ & 255.2 \\
\hline Transect 4 (D19) & $60 \mathrm{a}$ & -1.15 & -9.70 & $\mathrm{C} 3$ & 255.2 \\
\hline Transect 4 (D19) & $60 \mathrm{a}$ & -3.32 & -9.11 & $\mathrm{C} 4$ & 255.2 \\
\hline Transect 4 (D19) & $60 \mathrm{~B}$ & -5.10 & -9.09 & $\mathrm{C} 4$ & 255.2 \\
\hline Transect 4 (D19) & $60 \mathrm{~B}$ & -4.73 & -8.84 & $\mathrm{C} 4$ & 255.2 \\
\hline Transect 4 (D19) & $60 \mathrm{~B}$ & -4.15 & -9.18 & $\mathrm{C} 4$ & 255.2 \\
\hline Transect 4 (D19) & $60 \mathrm{~B}$ & -4.07 & -9.16 & $\mathrm{C} 4$ & 255.2 \\
\hline Transect 4 (D19) & $60 \mathrm{~B}$ & -2.90 & -9.06 & $\mathrm{C} 4$ & 255.2 \\
\hline Transect 4 (D19) & $60 a$ & -3.83 & -7.85 & M & 255.2 \\
\hline Transect 4 (D19) & $60 \mathrm{~B}$ & -5.04 & -9.17 & M & 255.2 \\
\hline Transect 4 (D19) & $60 \mathrm{~B}$ & -4.25 & -8.14 & $\mathrm{M}$ & 255.2 \\
\hline Transect 4 (D19) & $60 \mathrm{~B}$ & -3.61 & -8.58 & M & 255.2 \\
\hline Transect 4 (D19) & $60 \mathrm{~B}$ & -3.61 & -8.13 & M & 255.2 \\
\hline
\end{tabular}




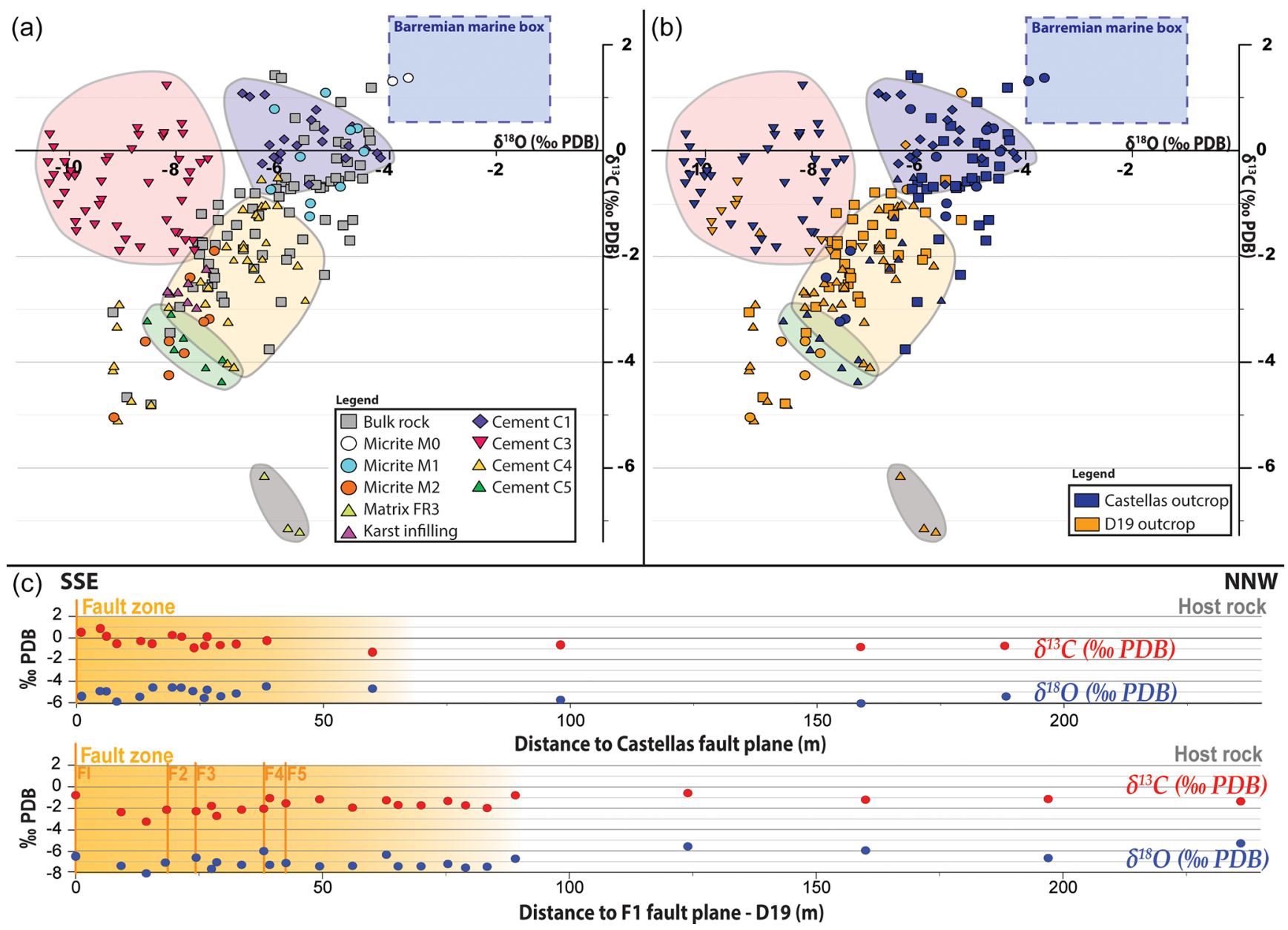

Figure 6. Isotopic values of $\delta^{13} \mathrm{C}$ and $\delta^{18} \mathrm{O}$ measured on bulk rock, cement phases, and micrite. Range values of "Urgonian marine box" from Moss and Tucker (1995) and Godet et al. (2006); (a) set of values sorted by the nature of diagenetic phases and (b) values sorted by the fault zone; (c) lateral evolution of $\delta^{13} \mathrm{C}$ and $\delta^{18} \mathrm{O}$ bulk isotopic values in Castellas (top) and in D19 (bottom) fault zones.

ii. cementation of $\mathrm{C} 1 \mathrm{a}$, partly filling intergranular porosity (Fig. 9B1b).

The micrite recrystallization strongly increased rock porosity due to enhanced microporosity (Fig. 9B1a). Resulting from this event, Urgonian carbonates formed a type III reservoir sensu Nelson (2001).

\subsubsection{Fault-related diagenesis - alteration of reservoir properties}

\section{Normal faulting-related diagenesis}

The Castellas Fault first nucleated during Durancian uplift (Aubert et al., 2019b; Matonti et al., 2012) affecting the host Urgonian carbonates. In porous granular media, fault nucleation mechanisms can lead to dilation processes (Fossen and Bale, 2007; Fossen and Rotevatn, 2016; Main et al., 2000; Wilkins et al., 2007; Zhu and Wong, 1997) under lowconfining pressure ( $<100 \mathrm{KPa}$; Alikarami and Torabi 2015).
Because this process leads to dilatancy, it increases the rock permeability (Alikarami and Torabi, 2015; Bernard et al., 2002) in the first stage of deformation bands development (Heiland et al., 2001; Lothe et al., 2002), enhancing fluid flows. Castellas Fault zone nucleated within a partially and dimly cemented host rock under low-confining pressure, in an extensional stress regime, at a depth $<1 \mathrm{~km}$ (Lamarche et al., 2012). Under these conditions, Barremian host rocks were likely characterized by mechanical and petrophysical properties close to porous granular media described above. Moreover, Micarelli et al. (2006) showed that during early stages of deformation, fault zones in carbonates have a hydraulic behavior comparable to deformation bands in carbonates. Hence, in the Urgonian carbonates of the La Fare area, dilatant processes occurred as an incipient fault mechanism and enhanced fluid circulations along the deformation bands. Fluid flows led to the cementation of C1b (Step 2 in Fig. 8). However, dilation bands were likely unstable, and grain collapse occurred swiftly after the beginning of the de- 


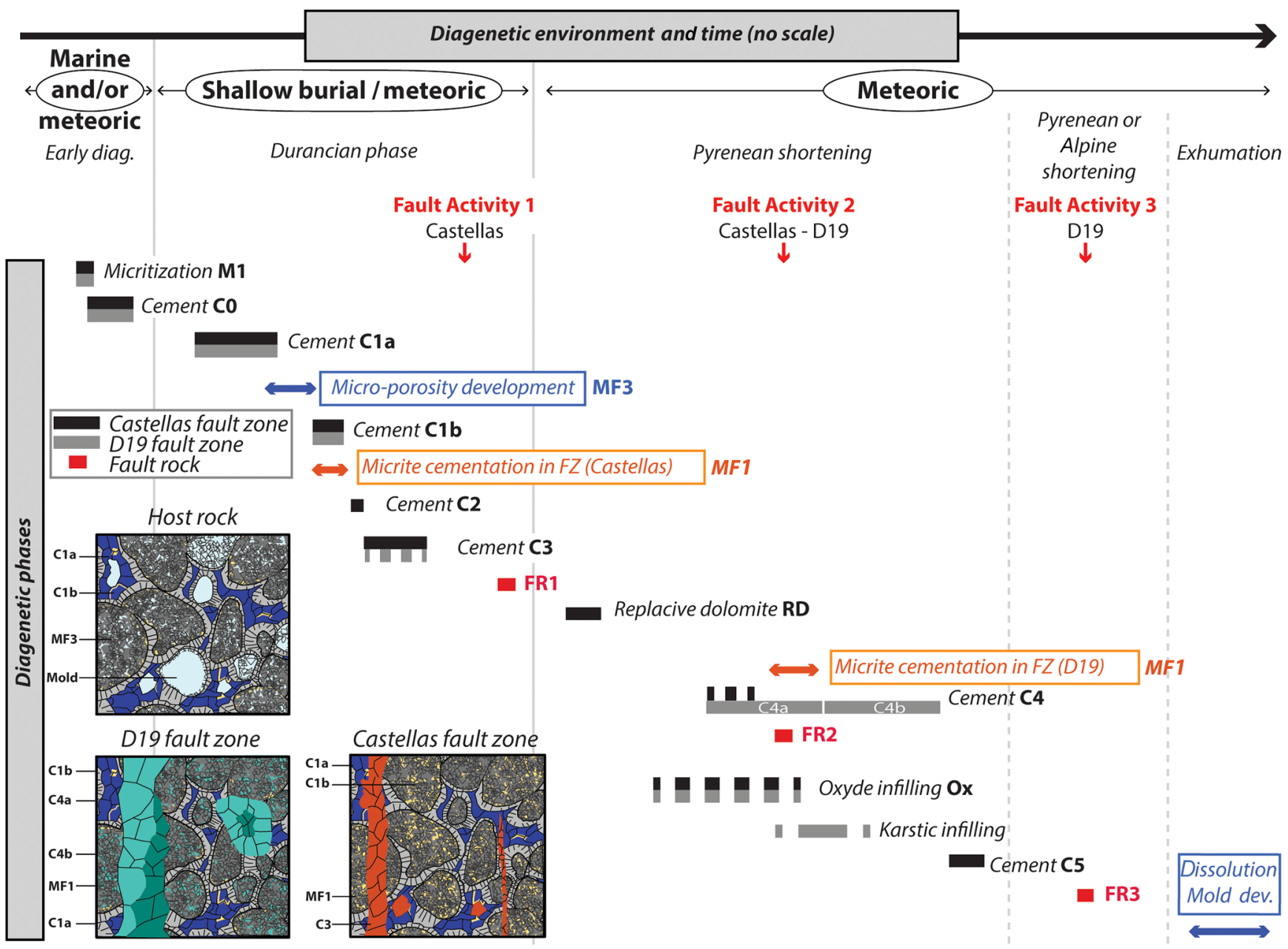

Figure 7. Paragenetic sequence of the both fault zones (black: Castellas; grey: D19) with microporosity development (blue), cementation (orange), and fault zone activation events (red).

formation due to an increase in the loading stress (Lothe et al., 2002). This could explain why $\mathrm{C} 1 \mathrm{~b}$ does not fill all intergranular porosity. Consequently, as all micritic grains in the fault zone are cemented by $\mathrm{Clb}$, the bulk isotopic measurements are strongly influenced by $\mathrm{C} 1$ cement isotopic values. This is the explanation why in transect 3 the bulk isotopic values $30 \mathrm{~m}$ apart from the fault (means of $-5.26 \%$ for $\delta^{18} \mathrm{O}$ and $-0.82 \%$ ofor $\delta^{13} \mathrm{C}$ ) are close to bulk isotopic values far from the fault plane $\left(188 \mathrm{~m} ;-5.37 \%\right.$ for $\delta^{18} \mathrm{O}$ and $-0.65 \%$ o for $\delta^{13} \mathrm{C}$, Fig. 6a). Dilation bands have also been described by Kaminskaite et al. (2019) in the San Vito Lo Capo carbonates grainstones (Sicily, Italy). These dilation bands also led to selective cementation of the carbonate rocks and to a microporosity decrease.

Cementation (C1a and $\mathrm{C} 1 \mathrm{~b})$ conferred a stiffer response of limestone to deformation, making it prone to deform through brittle structures (joints and veins), rather than via granular particulate flow (deformation bands). During the first stages of fault evolution in low-porosity limestones, intense fractur- ing of the fault zone predating fault core formation is known to increase fault permeability (Micarelli et al., 2006). In the studied faults, the first brittle event allowed Al-rich fluids to flow with fine-grained quartz grains in the incipient open fractures leading to precipitation of $\mathrm{C} 2$ cement (Step 3 in Fig. 8). The Urgonian facies of the studied area are composed of pure carbonates without siliciclastic input. Quartz grains and aluminum could have been reworked from surrounding formations. The rocks underlying the studied exposed Urgonian carbonates are limestones and dolostones. Albian and Aptian rocks are marly and sandy limestones, respectively (Anglada et al., 1977). Hence, Aptian layers are very likely to be the source of quartz. The fluids may have carried small grains of quartz from the Aptian sandy limestones via the fracture network. The $\mathrm{Al}$ enrichment of $\mathrm{C} 2$ could result from the erosion of Albian and Aptian deposits during the Durancian uplift (Guendon and Parron, 1985; Triat, 1982).

As the fault zone grew, new fracture sets formed, leading to new phase of calcite cementation (C3) in veins and 


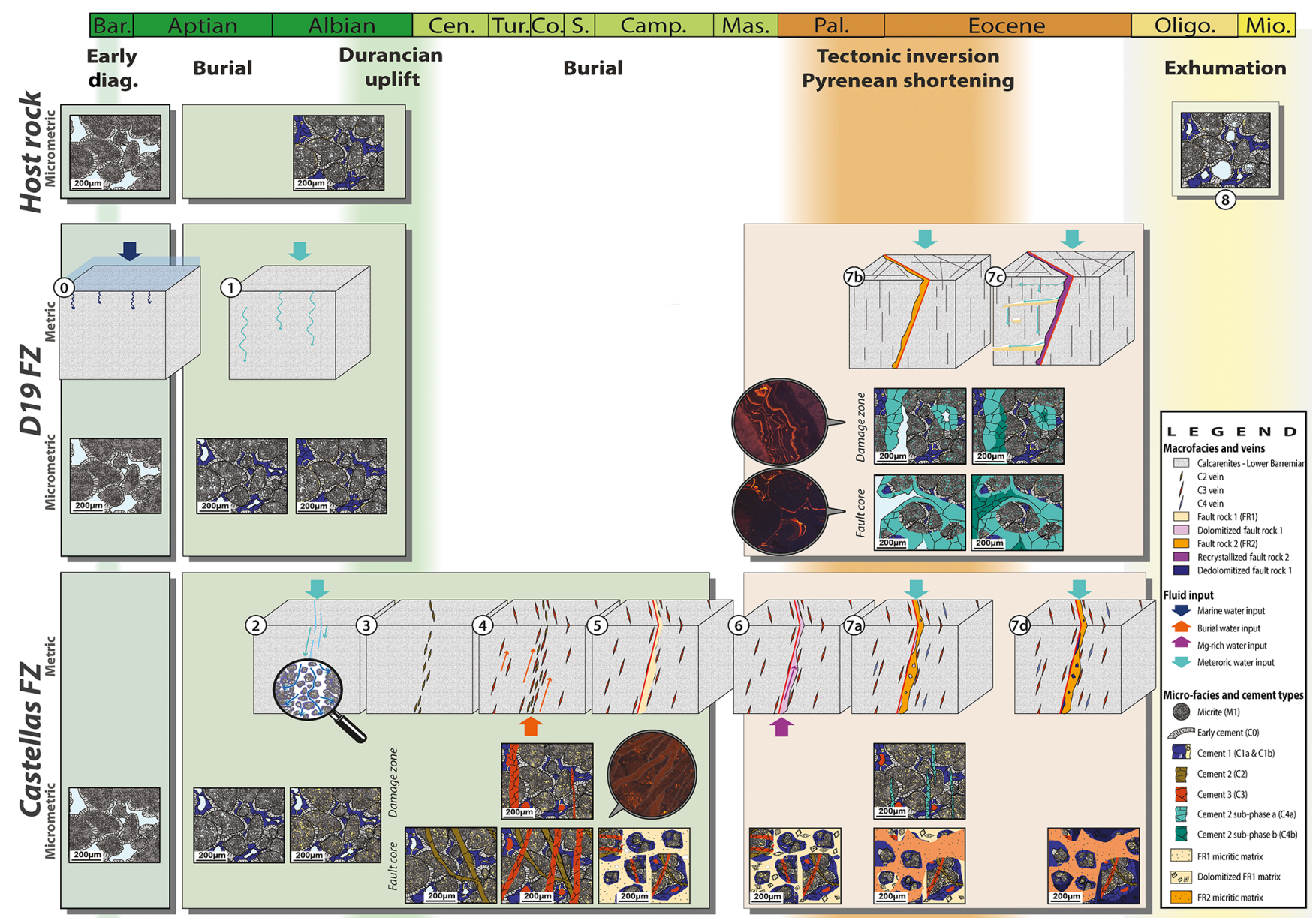

Figure 8. Diagenetic and geodynamic evolution since the Barremian of both fault zones and host rock at the metric and micrometric scale. Numbers 0 to 8 correspond to the Steps 0 to 8 (see text for description).

intergranular porosity (Step 4 in Fig. 8). The $\delta^{18} \mathrm{O}$ isotopic values of $\mathrm{C} 3$ range from $-10.40 \%$ to $-6.73 \%$ with $\delta^{13} \mathrm{C}$ values between $-2.09 \%$ and $+1.22 \%$. As $\mathrm{C} 3$ cementation occurred during the Durancian uplift and denudation, it most probably did not cement in deep burial conditions (maximum depth of $500 \mathrm{~m}$; Fig. 9C4). The negative $\delta^{13} \mathrm{C}$ values tend to corroborate the hypothesis of cementation induced by meteoric fluids rather than marine ones. Hence, C3 would correspond to a shallow-burial/meteoric cementation phase. Due to this cementation, rocks in this zone tightened with porosity down to $<5 \%$. The porosity has not changed since this event (Fig. 9B5). This porosity reduction due to cementation has also been observed in other cases of brittle-dilatant faults (Agosta et al., 2007; Celico et al., 2006; Gaviglio et al., 2009; Mozley and Goodwin, 1995). Following this, the fault zone was a barrier to fluid flow, leading to a reservoir compartmentalization. Fluids responsible for precipitation of $\mathrm{C} 3$ cement also occurred along fracture clusters of the D19 sector and led to vein formation.

In a later stage, the fault core formed, and the fault plane sensu-stricto developed, leading to FR1 breccia with a per- meable matrix with quartz grains $>100 \mu \mathrm{m}$ in size (Step 5 in Fig. 8). These grains came either from silica found inside C2 cement described above or from Aptian overlying rocks. Silica crystals in $\mathrm{C} 2$ veins are scarce and smaller than $10 \mu \mathrm{m}$. Thus, quartz grains may rather come from Aptian rocks like the ones found in $\mathrm{C} 2$ veins. The presence of Aptian quartz in the fault core proves that the Castellas Fault affected also Aptian rocks, which were later eroded during the Durancian uplift. According to this, the fault activity occurred before total erosion of Aptian rocks. Uncemented breccias within the fault core formed good fluid pathways (Billi et al., 2008; Delle Piane et al., 2016). In the studied fault, formation of FR1 breccia allowed the fault core to act as a drain. However, the cemented surrounding host rocks constrained the lateral extent of the drainage area of this high-permeability conduit. Uncemented breccias acting as good across- and along-fluid pathways were also described on Apennine carbonate formations within fault cores of strike-slip and extensional faults (Billi et al., 2003, 2008; Storti et al., 2003). 


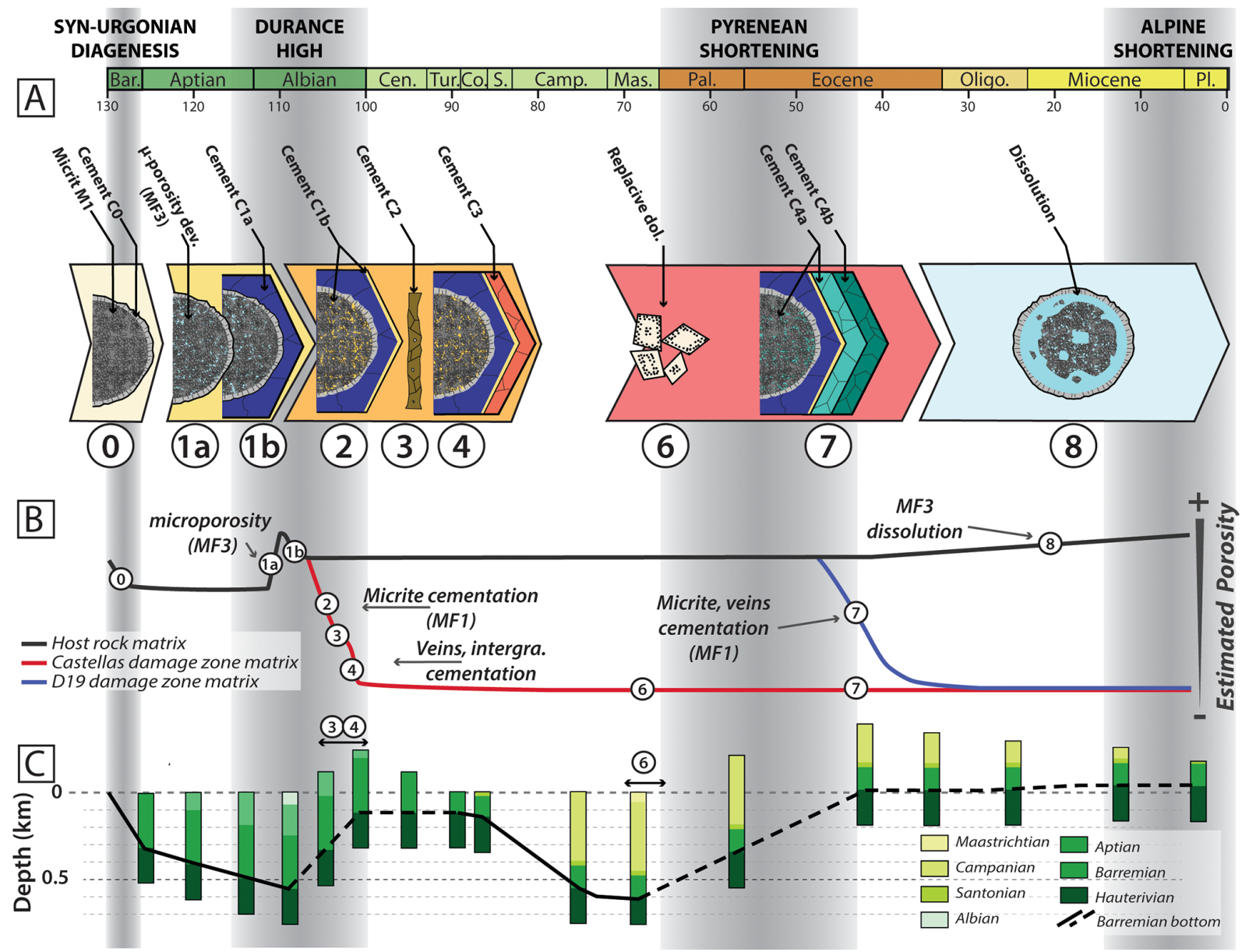

Figure 9. Evolution of reservoir properties. (A) different cementation phases; numbers 0 to 8 correspond to the Steps 0 to 8 (see text for description), (B) relative porosity evolution of the host rock and the two fault zones; (C) burial/uplift curve of Barremian basement (modified from Matonti et al. 2012).

\section{Tectonic inversion - Castellas Fault-related dolomitization}

At the onset of the Pyrenean shortening, compressive stresses led to underground water upwelling through the permeable fault core. This fluid flow triggered the dolomitization of the FR1 matrix (Step 6 in Fig. 8). This matrix-selective dolomitization could have been favored by several factors:

i. The matrix has higher permeability than cemented clasts with a smaller grain size, hence a higher grain surface area (Machel, 2004).

ii. This type of upwelling fluids, so-called "squeegeetype", are short-lived processes (Buschkuehle and Machel, 2002; Deming et al., 1990; Dorobek, 1989; Machel et al., 2000) not favorable for massive dolomitization.

iii. Low-temperature fluids, under $50-80^{\circ} \mathrm{C}$, enabled the preservation of FR1 clast initial structure. Contrarily, high-temperature dolomitization tends to be destructive (Machel, 2004).

iv. The tight surrounding host rock constrained Mg-rich fluid circulation to the fault core domain.

Gisquet et al. (2013) noticed similar fault-related replacive dolomitization phase in the Etoile massif, $23 \mathrm{~km}$ southeast of the studied zones. They linked the dolomitization to contractional stress regime during the early (Late Cretaceous) Pyrenean shortening. According to these authors, the tectonic stress led to low-temperature upwelling fluids likely $\mathrm{Mg}$ enriched by the dissolution of underlying Jurassic dolomites. The Jurassic dolomites also occur in La Fare anticline. Since the fluids leading to dolomitization of the fault core were low temperature and since dolomites occur underground, it is possible that the dolomitization in La Fare and in the Etoile massif were similar and synchronous. Matrix dolomitization can increase intercrystalline and/or interparticle porosity by up to $13 \%$, but the later dolomite overgrowth reduces the porosity and permeability (Lucia, 2004; Machel, 2004; 
Saller and Henderson, 2001). Hence, in the first stages of dolomitization, the fault core was an important drain. After the growth of dolomite crystals, the fault core turned into a barrier (Fig. 9B6 and C6)

\section{Sinistral tectonic inversion - meteoric alteration of reservoir properties}

The ongoing tectonic inversion with increasing compressive stresses eventually led to the Castellas Fault sinistral reactivation and to the onset of the D19 Fault zone (Aubert et al., 2019b). Aubert et al. (2019a) has shown that this compression reactivated the pre-existing early $30^{\circ} \mathrm{N}$ background fractures (Step 7 in Fig. 8). This tectonic event formed FR2 in fault cores but with specific diagenetic consequences. In the D19 Fault zone, the fault nucleation and reactivation of background fractures led to plurimetric to kilometric fault surfaces with a permeable fault rock acting as drains and localizing the fluid flow (Aubert et al., 2019a). This fluid flow witnessed by the cementation of $\mathrm{C} 4 \mathrm{a}$ and $\mathrm{C} 4 \mathrm{~b}$ in veins and micritized grains (MF1, Step 7c in Fig. 8), led to a strong porosity decrease in the fault zone (Fig. 9B7 and C7). However, not all fractures were cemented by $\mathrm{C} 4$, so that fracture porosity/permeability was still partially preserved. Therefore, the D19 Fault zone became a type I reservoir sensu Nelson (2001) with a very low matrix porosity/permeability and high fracture-related secondary permeability (Aubert et al., 2019a).

Along F2, successive fluids gave rise to karsts, karstic infilling, and dissolution/cementation processes of the FR2 matrix (Step 7c in Fig. 8). Then, FR2 was sealed by C4 cementation. Isotopic values of $\mathrm{C} 4$ cement (from $-9.2 \%$ o to $-6.1 \%$ for $\delta^{18} \mathrm{O}$ and from $-5.01 \%$ to $-1.0 \%$ ofor $\delta^{13} \mathrm{C}$ ) highlight the strong influence of meteoric fluids. This is coherent with the occurrence of karstic infilling due to fluid circulations in vadose zone, with alternating dissolution and cementation (Swart, 2015). However, the positive covariance between $\delta^{18} \mathrm{O}$ and $\delta^{13} \mathrm{C}$ of $\mathrm{C} 4$ suggests mixed fluids (Allan and Matthews, 1982) of meteoric water and burial or marine water.

In the Castellas Fault zone, the host rocks are slightly impacted by these meteoric fluid circulations. Yet, some veins filled with $\mathrm{C} 4 \mathrm{a}$ cement occur along transect 2 and 3 (Step 7a in Fig. 8). Two samples have enriched $\delta^{18} \mathrm{O}$ and $\delta^{13} \mathrm{C}$ isotopic values (respective means of $-6.25 \%$ and $-4.20 \%$ for $\delta^{18} \mathrm{O} ;-0.64$ and $-0.09 \%$ for $\delta^{18} \mathrm{O}$ ) similar to $\mathrm{C} 1$ cement (Fig. 6a). This indicates that $\mathrm{C} 4$ cement in the Castellas Fault zone was precocious in comparison to $\mathrm{D} 19$. $\mathrm{C} 4$ cement in the Castellas area is restricted to transect 2 . Transect 2 crosscuts the Castellas Fault along a relay zone (Fig. 2A). Relay or linkage zones occur where two fault segments overlap each other during fault grow (Kim et al., 2004; Long and Imber, 2011; Walsh et al., 1999, 2003). Consequently, the fault complexity, the fracture intensity and the fracture-strike range are increased (Kim et al., 2004; Sibson, 1996). This process in the studied area resulted in a well-connected fracture network that increased the permeability and favored local fluid circulations. In transect 2 , the increase in the local permeability in the relay zone enhanced fluid flow related to $\mathrm{C} 4$ cement. The relay zones along the Castellas Fault and their consequences on the fracture permeability are, therefore, responsible for this local cementation event. On the contrary, cementation in the D19 Fault zone is linked to the highly permeable fault surfaces, which acted as drains (Aubert et al., 2019a). This implies that the cementation occurred only after the development of the fault surface. In the case of Castellas, the relay zone was already present, inherited from the former extensional activity, allowing early $\mathrm{C} 4$ fluid to flow through the fault zone. This, in addition, explains why the early $\mathrm{C} 4$ cementation has not been recorded in D19 Fault zone. The C4 cementation in transect 2 reduced porosity to less than $8 \%$ on a wider zone $(>60 \mathrm{~m})$ than in both other transects (transect $1 \approx 30 \mathrm{~m}$, transect $3>40 \mathrm{~m}$ ).

The reactivation of the Castellas Fault formed a new fracture network that locally triggered the fracture connectivity and permeability. The Castellas Fault zone formed a type I reservoir (Nelson, 2001), but lateral variation in the fracture network implies lateral variations in the hydraulic properties. Thus, the fault zone was both a drain and a barrier (Matonti et al., 2012). In this case, the most appropriate concept would be a sieve, because in this analogy, it is synchronously closed in places and open in other places.

After these events, the matrix of the Castellas Fault core was dedolomitized (FR1) in relation to cementation C5 (Step $7 \mathrm{~d}$ in Fig. 8). The C5 cement isotope values (mean of $-7.49 \%$ or for $\delta^{18} \mathrm{O}$ and $-4.01 \%$ for $\delta^{13} \mathrm{C}$ ) are comprised within $\mathrm{C} 4$ positive covariance between $\delta^{18} \mathrm{O}$ and $\delta^{13} \mathrm{C}$. This indicates a continuity between $\mathrm{C} 4$ and $\mathrm{C} 5$ fluid flows. The measurements with the SEM revealed a lack of $\mathrm{Mg}$ in the matrix indicating that $\mathrm{C} 5$ totally recrystallized the replacive dolomite. Following this dedolomitization phase, no additional diagenetic event is recorded in the Castellas Fault zone.

A late Pyrenean to Alpine compression reactivated the D19 Fault zone that formed the new fault rock FR3. The matrix of this fault rock has very low $\delta^{13} \mathrm{C}$ isotopic values (mean of $-6.83 \%$ ) indicating an organic matter input (Swart, 2015). This implies fluids percolating soils, as results from a near-surface fluid circulation. We deduce that the D19 Fault was lately reactivated after the folding of the La Fare anticline. There is no such cementation with similar isotope values in the fault zone, meaning that fluids and cements did not alter the fault zone diagenetic properties.

Eventually, the late exhumation of the Urgonian carbonate host rocks led to flows inducing dissolution of MF3 grains in the host rock. This phase produced the moldic porosity and increased the porosity/permeability (Step 8 in Fig. 9B and C). These fluids, however, did not affect fault zones. 

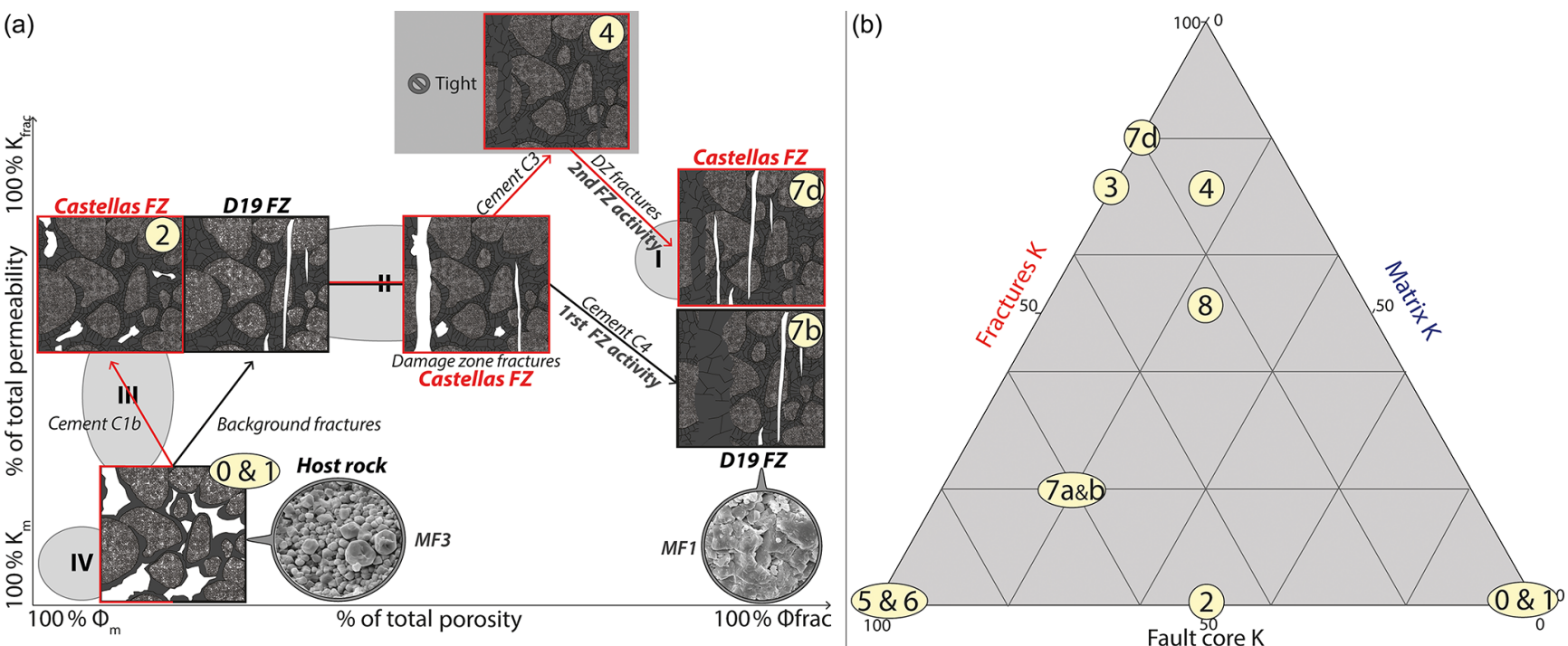

Figure 10. Castellas and D19 Fault zone reservoir properties evolution. (a) Evolution of permeability and porosity taking into account fault zone fractures and matrix after Nelson (2001) and (b) triangle diagram of permeability evolution with three components: matrix, fractures, and fault core. Numbers 1 to 8 correspond to the Steps 1 to 8 (see text for description). K: permeability; $\Phi$ : porosity; FZ: fault zone; DZ: damage zone; MF1 and MF3: micrite micro-fabric.

\subsection{Evolution of fault zones reservoir properties}

The host rock presents a monophasic evolution and switch from a type IV reservoir where the matrix provided storage and flow, to a type III reservoir where fractures behave as pathways towards fluid flow, but the production mainly comes from the matrix (Nelson, 2001, Fig. 10a). The fault zones present a more complex polyphasic evolution than the host rock. Indeed, their reservoir properties evolved from a type IV reservoir corresponding to the host rock to a type I reservoir where fractures provide both storage and flow pathways (Nelson, 2001, Fig. 10a). Both fault zones present slight differences. The Castellas Fault zone was completely tight soon after $\mathrm{C} 3$ cementation. Consequently, it did not fit to the Nelson reservoir-type classification. However, after fault core formation, the fault zone presents a high fault core permeability. In this study we propose a new approach with a triangle diagram taking into account fault core permeability to remove the flaws of this method (Fig. 10b). The percentage assigned to the fault core or to the matrix are qualitatively estimated. Further quantification could be evaluated, for instance, with the width of the fault core and damage zone domains or by estimating the fracture network volume. However, no recent study has provided such quantification. Thus, for the Castellas Fault zone, permeability evolves from a stage with exclusive contribution from the host rock permeability (100\% matrix; Step 0 in Fig. 10b) to a permeability due $50 \%$ to the matrix and $50 \%$ to the fault core during dilation band development (Step 2 in Fig. 10b). Thereafter, during the two fracture events permeability is mainly linked to fracturing (C2: $30 \%$ fault core, $70 \%$ fractures; $\mathrm{C} 3$ :
$15 \%$ fault core, $15 \%$ matrix, and $70 \%$ fractures; Steps 3 and 4 in Fig. 10b). Then, after fault core formation and during dolomitization event, permeability is solely provided by the fault core (Steps 6 and 7 in Fig. 10b). Lastly, after fault zone reactivation, the permeability is due $20 \%$ to the fault core and $80 \%$ to fractures (Step 7c in Fig. 10b). The D19 Fault zone permeability during its development was related for $20 \%$ to the matrix, $20 \%$ to the fractures, and $60 \%$ to the fault core (Steps 7a and 7b in Fig. 10b).

\section{Conclusions}

This study deciphered the diagenetic evolution of two fault zones and the impact on reservoir properties of both faults and host rock in the frame of the overall geodynamic context of the SE Basin. The main outcomes are as follows:

- Fault zones may have a complex diagenetic history, but most diagenetic phases occur during the nucleation of the fault. In the case of the Castellas Fault zone, the diagenetic imprint is mainly influenced by early diagenesis occurring along fractures and diffuse dilation zones prior to the proper fault plane nucleation. Regarding the D19 Fault zone, most of diagenetic alterations occurred just after fault onset in the first stage of its activity. In both cases, the cementation altered initial reservoir properties in the fault zone vicinity, switching from type III to type I during the first stages of fault development. Later fault reactivation slightly impacts matrix porosity/permeability. 
- Fault zones act as drains canalizing fluid flows in the beginning of their development. This induces fault zone cementation but preservation of host rock microporosity. This important fluid drainage is visible on the D19 outcrop where the flowing fluids led to dissolution/cementation of fault rock matrix and formed karsts.

- All diagenetic stages, including cementation and dolomitization, result from low-temperature fluids with important meteoric water input. These low-temperature fluid flows associated with the deformation and cementation types and the lack of mineralization specific to high-temperature fluids disprove any significant hydrothermal influence.

This regional study allows broader rules to be drawn for complex faults with polyphasic activity affecting granular carbonates at shallow burial conditions (Fig. 9).

- Under extensional context, fault nucleation can lead to the development of dilation bands acting as conduits for fluid flow. Carbonates are very sensitive to rock-fluid interactions. Thus, the onset of dilation bands triggers important diagenetic reactions that strongly alter local reservoir properties. During later fault zone development, the diagenesis depends on fault zone internal architecture.

- Fracture networks related to fault nucleation in granular carbonates form good fluid pathways before proper fault plane formation. However, in the case of pre-fractured carbonates, like D19 Fault zone, fault rocks appear early in fault cores. In these cases, fluids flowed preferentially within the permeable breccia rather than in the damage zone.

Data availability. Data can be accessed by request from any of the authors (https://doi.org/10.5281/zenodo.3903298, last access: 3 July 2020, Aubert et al., 2020).

Author contributions. IA, PL, and JL designed the study. IA, performed fieldwork and research. IA, PL and RS interpreted data. IA, $\mathrm{JL}$, and PL wrote the paper.

Competing interests. The authors declare that they have no conflict of interest.

Special issue statement. This article is part of the special issue "Faults, fractures, and fluid flow in the shallow crust". It is not associated with a conference.

Acknowledgements. We would like to thank Suzan Verdegaal, Lionel Marié, and Alain Tonetto for support they provide during this study. We grateful to Editor Kei Ogata and Fabrizio Agosta, Mattia Pizzati, and Eric Salomon who made critical suggestions to improve this paper.

Financial support. The authors greatly appreciate APIC Cerege, whose financial support enabled the isotopic measurements to be made.

Review statement. This paper was edited by Kei Ogata and reviewed by Eric Salomon, Mattia Pizzati, and one anonymous referee.

\section{References}

Agosta, F., Prasad, M., and Aydin, A.: Physical properties of carbonate fault rocks, Fucino Basin (Central Italy): implications for fault seal in platform carbonates, Geofluids, 7, 19-32, https://doi.org/10.1111/j.1468-8123.2006.00158.x, 2007.

Agosta, F., Mulch, A., Chamberlain, P., and Aydin, A.: Geochemical traces of CO2-rich fluid flow along normal faults in central Italy, Geophys. J. Int., 174, 1074-1096, https://doi.org/10.1111/j.1365-246X.2008.03792.x, 2008.

Agosta, F., Alessandroni, M., Antonellini, M., Tondi, E., and Giorgioni, M.: From fractures to flow: A field-based quantitative analysis of an outcropping carbonate reservoir, Tectonophysics, 490, 197-213, https://doi.org/10.1016/j.tecto.2010.05.005, 2010.

Agosta, F., Ruano, P., Rustichelli, A., Tondi, E., Galindo-Zaldívar, J., and Sanz de Galdeano, C.: Inner structure and deformation mechanisms of normal faults in conglomerates and carbonate grainstones (Granada Basin, Betic Cordillera, Spain): Inferences on fault permeability, J. Struct. Geol., 45, 4-20, https://doi.org/10.1016/j.jsg.2012.04.003, 2012.

Alikarami, R. and Torabi, A.: Geomechanics for Energy and the Environment Micro-texture and petrophysical properties of dilation and compaction shear bands in sand, Geomech. Energy Envir., 3, 1-10, https://doi.org/10.1016/j.gete.2015.06.001, 2015.

Allan, J. R. and Matthews, R. K.: Isotope signatures associated with early meteoric diagenesis, Sedimentology, 29, 797-817, https://doi.org/10.1111/j.1365-3091.1982.tb00085.x, 1982.

Allmendinger, R. W., Cardozo, N., and Fisher, D. M.: Structural geology algorithms: Vectors and tensors, Cambridge Univ. Press, 1-289, 9781107012, https://doi.org/10.1017/CBO9780511920202, 2013.

Anglada, R., Arlhac, P., Catzigras, F., Colomb, E., Damiani, L., Durand, J. P., Durozoy, G., Guieu, G., Masse, J. P., Nury, D., Philip, J., Rouire, J., Rousset, C., Roux, R. M., and Blanc, J. J.: Notice explicative, Carte géologique de la France a 1/50 000, Martigues - Marseille, 51 pp., 1977.

Aubert, I., Lamarche, J., and Léonide, P.: Deciphering background fractures from damage fractures in fault zones and their effect on reservoir properties in microporous carbonates (Urgonian limestones, SE France), Pet. Geosci., 25, 443, https://doi.org/10.1144/petgeo2019-010, 2019a.

Aubert, I., Lamarche, J., Richard, P., and Léonide, P.: Imbricated Structure and Hydraulic Path Induced by Strike Slip Reactiva- 
tion of a Normal Fault in Carbonates, in Fifth International Conference on Fault and Top Seals, p. 4., 2019b.

Aubert, I., Léonide, P., Lamarche, J., and Salardon, R.: Carbon and oxygen isotope values of bulk carbonates for Castellas Fault zone and D19 fault zones, La Fare anticline (SE France), https://doi.org/10.5281/zenodo.3903298, 2020.

Bense, V. F., Gleeson, T., Loveless, S. E., Bour, O., and Scibek, J.: Fault zone hydrogeology, Earth-Sci. Rev., 127, 171-192, https://doi.org/10.1016/j.earscirev.2013.09.008, 2013.

Bernard, X. D., Eichhubl, P., and Aydin, A.: Dilation bands?: A new form of localized failure in granular media, 29, 1-4, https://doi.org/10.1029/2002GL015966, 2002.

Besson, D.: Architecture du bassin rhodano-provençal miocène (Alpes, SE France): relations entre déformation, physiographie et sédimentation dans un bassin molassique d'avant-pays, Ecole des Mines, Paris, 450 pp., 2005.

Bestani, L.: Géométrie et cinématique de l'avant-pays provençal: Modélisation par coupes équilibrées dans une zone à tectonique polyphasée, Aix-Marseille University, 246 pp., 2015.

Bestani, L., Espurt, N., Lamarche, J., Bellier, O., and Hollender, F.: Reconstruction of the Provence Chain evolution, Southeastern France, Tectonics, 35, 1506-1525, https://doi.org/10.1002/2016TC004115, 2016.

Billi, A., Salvini, F., and Storti, F.: The damage zone-fault core transition in carbonate rocks: Implications for fault growth, structure and permeability, J. Struct. Geol., 25, 1779-1794, https://doi.org/10.1016/S0191-8141(03)00037-3, 2003.

Billi, A., Primavera, P., Soligo, M., and Tuccimei, P.: Minimal mass transfer across dolomitic granular fault cores, Geochem. Geophys., 9, ISSN 1525-2027, https://doi.org/10.1029/2007GC001752, 2008.

Borgomano, J., Masse, J., Maskiry, S. A., Borgomano, J., and International, S.: The lower Aptian Shuaiba carbonate outcrops in Jebel Akhdar, northern Oman: Impact on static modeling for Shuaiba petroleum reservoirs, Bull. Am. Assoc. Pet. Geol., 9, 1513-1529, https://doi.org/10.1306/61EEDCE2-173E-11D78645000102C1865D, 2002.

Borgomano, J., Masse, J. P., Fenerci-Masse, M., and Fournier, F.: Petrophysics of lower cretaceous platform carbonate outcrops in provence (SE France): Implications for carbonate reservoir characterisation, J. Pet. Geol., 36, 5-41, https://doi.org/10.1111/jpg.12540, 2013.

Bruna, P., Guglielmi, Y., Viseur, S., Lamarche, J., and Bildstein, O.: Coupling fracture facies with in-situ permeability measurements to generate stochastic simulations of tight carbonate aquifer properties: Example from the Lower Cretaceous aquifer, Northern Provence, SE France, J. Hydrol., 529, 737-753, https://doi.org/10.1016/j.jhydrol.2015.08.054, 2015.

Buschkuehle, B. E. and Machel, H. G.: Diagenesis and paleo fluid flow in the Devonian Southesk-Cairn carbonate complex in Alberta, Canada, Mar. Pet. Geol., 19, 219-227, https://doi.org/10.1016/S0264-8172(02)00014-4, 2002.

Caine, J. S., Evans, J. P., and Forster, C. B.: Fault zone architechture and permeability structure, Geology, 24, 1025-1028, https://doi.org/10.1130/0091-7613(1996)024<1025, 1996.

Cardozo, N. and Allmendinger, N. W.: Sperical projections with OSXStereonets, Comput. Geosci., 51, 193-205, https://doi.org/10.1016/j.cageo.2012.07.021, 2013.
Celico, F., Petrella, E., and Celico, P.: Hydrogeological behaviour of some fault zones in a carbonate aquifer of Southern Italy: An experimentally based model, Terra Nov., 18, 308-313, https://doi.org/10.1111/j.1365-3121.2006.00694.x, 2006.

Champion, C., Choukroune, P., and Clauzon, G.: La déformation post-miocène en provence occidentale, Geodin. Acta, 13, 67-85, https://doi.org/10.1080/09853111.2000.11105365, 2000.

Chester, F. M. and Logan, J. M.: Implications for Mechanical Properties of Brittle Faults from Observations of the Punchbowl Fault Zone, California, Pure Appl. Geohys., 124, 79, https://doi.org/10.1007/BF00875720, 1986.

Chester, F. M. and Logan, J. M.: Composite planar fabric of gouge from the Punchbowl Fault, California, J. Struct. Geol., 9, https://doi.org/10.1016/0191-8141(87)90147-7, 1987.

Delle Piane, C., Giwelli, A., Clennell, M. Ben, Esteban, L., Nogueira Kiewiet, M. C. D., Kiewiet, L., Kager, S., and Raimon, J.: Frictional and hydraulic behaviour of carbonate fault gouge during fault reactivation - An experimental study, Tectonophysics, 690, 21-34, https://doi.org/10.1016/j.tecto.2016.07.011, 2016.

Deming, D., Nunn, A., and Evans, D. G.: Thermal Effects of Compaction-Driven Groundwater Flow, J. Geophys. Res.-Sol. Ea, 95, 6669-6683, https://doi.org/10.1029/JB095iB05p06669, 1990.

Demory, F. R., Conesa, G. I., Oudet, J. U., Mansouri, H. A., and Münch, P. H.: Magnetostratigraphy and paleoenvironments in shallow-water carbonates: The Oligocene-Miocene sediments of the northern margin of the Liguro- Provençal basin (West Marseille, southeastern France), Bull. Soc. Géol. Fr., 1, 37-55, https://doi.org/10.2113/gssgfbull.182.1.37, 2011.

Deville de Periere, M., Durlet, C., Vennin, E., Lambert, L., Bourillot, R., Caline, B., and Poli, E.: Morphometry of micrite particles in cretaceous microporous limestones of the middle east: Influence on reservoir properties, Mar. Pet. Geol., 28, 1727-1750, https://doi.org/10.1016/j.marpetgeo.2011.05.002, 2011.

Deville de Periere, M., Durlet, C., Vennin, E., Caline, B., Boichard, R., and Meyer, A.: Influence of a major exposure surface on the development of microporous micritic limestones - Example of the Upper Mishrif Formation (Cenomanian) of the Middle East, Sediment. Geol., 353, 96-113, https://doi.org/10.1016/j.sedgeo.2017.03.005, 2017.

Dorobek, S.: migration of erogenic fluids through the SiluroDevonian Helderberg Group during late Paleozoic deformation: constraints on fluid sources and implications for thermal histories of sedimentary basins presence, Tectonophysics, 159, 25-45, https://doi.org/10.1016/0040-1951(89)90168-6, 1989.

Eltom, H. A., Gonzalez, L. A., Hasiotis, S. T., Rankey, E. C., and Cantrell, D. L.: Paleogeographic and paleooceanographic influences on carbon isotope signatures: Implications for global and regional correlation, Middle-Upper Jurassic of Saudi Arabia, Sediment. Geol., 364, 89-102, https://doi.org/10.1016/j.sedgeo.2017.12.011, 2018.

Espurt, N., Hippolyte, J. C., Saillard, M., and Bellier, O.: Geometry and kinematic evolution of a long-living foreland structure inferred from field data and cross section balancing, the SainteVictoire System, Provence, France, Tectonics, 31, TC4021, https://doi.org/10.1029/2011TC002988, 2012.

Evans, J. P., Forster, C. B., and Goddard, J. V.: Permeability of fault-related rocks, and implications for hydraulic 
structure of fault zones, J. Struct. Geol., 19, 1393-1404, https://doi.org/10.1016/S0191-8141(97)00057-6, 1997.

Ferraro, F., Agosta, F., Ukar, E., Grieco, D. S., Cavalcante, F., Belviso, C., and Prosser, G.: Structural diagenesis of carbonate fault rocks exhumed from shallow crustal depths: An example from the central-southern Apennines, Italy, J. Struct. Geol., 122, 58-80, https://doi.org/10.1016/j.jsg.2019.02.008, 2019.

Florida, S., Maliva, R. G., Missimer, T. M., Clayton, E. A., and Dickson, J., A. D.: Diagenesis and porosity preservation in Eocene microporous limestones, Sediment. Geol., 217, 85-94, https://doi.org/10.1016/j.sedgeo.2009.03.011, 2009.

Ford, M., Duchene, S., Gasquet, D., and Vanderhaeghe, O.: Two-phase orogenic convergence in the external and internal SW Alps, J. Geol. Soc. Lond., 163, 815-826, https://doi.org/10.1144/0016-76492005-034, 2006.

Fossen, H. and Bale, A.: Deformation bands and their influence on fluid flow, 12, 1685-1700, https://doi.org/10.1306/07300706146, 2007.

Fossen, H. and Rotevatn, A.: Fault linkage and relay structures in extensional settings - A review, Earth Sci. Rev., 154, 14-28, https://doi.org/10.1016/j.earscirev.2015.11.014, 2016.

Fouke, B. W., Everts, A. W., Zwart, E. W., and Schlager, W.: Subaerial exposure unconformities on the Vercors carbonate platform (SE France) and their sequence stratigraphic significance, Geol. Soc. Lond., 104, 295-319, 1996.

Fournier, F. and Borgomano, J.: Critical porosity and elastic properties of microporous mixed carbonate-siliciclastic rocks, Geophysics, 74, 93-109, https://doi.org/10.1190/1.3043727, 2009.

Fournier, F., Léonide, P., Biscarrat, K., Gallois, A., Borgomano, J., and Foubert, A.: Elastic properties of microporous cemented grainstones, Geophysics, 76, 211-226, https://doi.org/10.1190/geo2011-0047.1, 2011.

Gattacceca, J., Deino, A., Rizzo, R., Jones, D. S., Henry, B., Beaudoin, B., and Vadeboin, F.: Miocene rotation of Sardinia: New paleomagnetic and geochronological constraints and geodynamic implications, Earth Planet. Sc. Lett., 258, 359-377, https://doi.org/10.1016/j.epsl.2007.02.003, 2007.

Gaviglio, P., Bekri, S., Vandycke, S., Adler, P. M., Schroeder, C., Bergerat, F., Darquennes, A., and Coulon, M.: Faulting and deformation in chalk, J. Struct. Geol., 31, 194-207, https://doi.org/10.1016/j.jsg.2008.11.011, 2009.

Gisquet, F., Lamarche, J., Floquet, M., Borgomano, J., Masse, J. P., and Caline, B.: Three-dimensional structural model of composite dolomite bodies in folded area (upper Jurassic of the Etoile massif, southeastern France), Am. Assoc. Pet. Geol. Bull., 97, 1477-1501, https://doi.org/10.1306/04021312016, 2013.

Godet, A., Bodin, S., Föllmi, K. B., Vermeulen, J., Gardin, S., Fiet, N., Adatte, T., Berner, Z., Stüben, D., and van de Schootbrugge, B.: Evolution of the marine stable carbon-isotope record during the early Cretaceous: A focus on the late Hauterivian and Barremian in the Tethyan realm, Earth Planet. Sc. Lett., 242, 254 271, https://doi.org/10.1016/j.epsl.2005.12.011, 2006.

Guendon, J.-L. and Parron, C.: Les phenomenes karstiques dans les processus de la bauxitisation sur substrat carbonate. Exemple de gisement du sud est de la France, Ann. la Société Géologique Belgique, 108, 85-92, 1985.

Guieu, G.: Un exemple de tectonique tangentielle: l'évolution du cadre montagneux de Marseille, Bull. la Société Géologique Fr., 7, 610-630, 1967.
Guyonnet-Benaize, C., Lamarche, J., Masse, J. P., Villeneuve, M., and Viseur, S.: 3D structural modelling of small-deformations in poly-phase faults pattern. Application to the Mid-Cretaceous Durance uplift, Provence (SE France), J. Geodyn., 50, 81-93, https://doi.org/10.1016/j.jog.2010.03.003, 2010.

Hammond, K. J. and Evans, J. P.: Geochemistry, mineralization, structure, and permeability of a normal- fault zone, Casino mine, Alligator Ridge district, north central Nevada, J. Struct. Geol., 25, 717-736, https://doi.org/10.1016/S0191-8141(02)00060-3, 2003.

Heiland, J., Raab, S., and Potsdam, G.: Experimental Investigation of the Influence of Differential Stress on Permeability of a Lower Permian (Rotliegend) Sandstone Deformed in the Brittle Deformation, Phys. Chem. Earth, 26, 33-38, https://doi.org/10.1016/S1464-1895(01)00019-9, 2001.

Hodson, K. R., Crider, J. G., and Huntington, K. W.: Temperature and composition of carbonate cements record early structural control on cementation in a nascent deformation band fault zone: Moab Fault, Utah, USA, Tectonophysics, 690, 240-252, https://doi.org/10.1016/j.tecto.2016.04.032, 2016.

Hollis, C., Vahrenkamp, V., Tull, S., Mookerjee, A., and Taberner, C.: Pore system characterisation in heterogeneous carbonates: An alternative approach to widely-used rock-typing methodologies, Mar. Pet. Geol., 27, 772-793, https://doi.org/10.1016/j.marpetgeo.2009.12.002, 2010.

Kaminskaite, I., Fisher, Q. J., and Michie, E. A. H.: Microstructure and petrophysical properties of deformation bands in high porosity carbonates, J. Struct. Geol., 119, 61-80, https://doi.org/10.1016/j.jsg.2018.12.001, 2019.

Kim, Y. S., Peacock, D. C. P., and Sanderson, D. J.: Fault damage zones, J. Struct. Geol., 26, 503-517, https://doi.org/10.1016/j.jsg.2003.08.002, 2004.

Knipe, R. J.: The influence of fault zone processes and diagenesis on fluid flow, Diagenes, available at: http://archives.datapages.com/ data/specpubs/resmi1/data/a067/a067/0001/0100/0135.htm,

basin Dev. AAPG Stud. Geol., Vol. 36, edited by: Horbury, A. D. and Robinson, A. G., 135-148, 1993.

Knipe, R. J., Jones, G., and Fisher, Q. J.: Faulting, fault sealing and fluid flow in hydrocarbon reservoirs: an introduction, Geol. Soc. Lond, 147, NP LP-NP, https://doi.org/10.1144/GSL.SP.1998.147.01.21, 1998.

Lamarche, J., Lavenu, A. P. C., Gauthier, B. D. M., Guglielmi, Y., and Jayet, O.: Relationships between fracture patterns, geodynamics and mechanical stratigraphy in Carbonates (South-East Basin, France), Tectonophysics, 581, 231-245, https://doi.org/10.1016/j.tecto.2012.06.042, 2012.

Lambert, L., Durlet, C., Loreau, J. P., and Marnier, G.: Burial dissolution of micrite in Middle East carbonate reservoirs (JurassicCretaceous): Keys for recognition and timing, Mar. Pet. Geol., 23, 79-92, https://doi.org/10.1016/j.marpetgeo.2005.04.003, 2006.

Laubach, S. E., Eichhubl, P., Hilgers, C., and Lander, R. H.: Structural diagenesis, J. Struct. Geol., 32, 1866-1872, https://doi.org/10.1016/j.jsg.2010.10.001, 2010.

Lavenu, A. P. C., Lamarche, J., Gallois, A., and Gauthier, B. D. M.: Tectonic versus diagenetic origin of fractures in a naturally fractured carbonate reservoir analog Nerthe anticline, Southeastern France, Am. Assoc. Pet. Geol. Bull., 97, 2207-2232, https://doi.org/10.1306/04041312225, 2013. 
Le Pichon, X., Bergerat, F., and Roulet, M.-J.: Plate kinematics and tectonics leading to the Alpine belt formation; A new analysis, Geol. Soc. Am., 218, 111-131, https://doi.org/10.1130/SPE218p111, 1988.

Léonide, P., Borgomano, J., Masse, J., and Doublet, S.: Relation between stratigraphic architecture and multi-scale heterogeneities in carbonate platforms: The Barremian - lower Aptian of the Monts de Vaucluse, SE France, Sediment. Geol., 265, 87-109, https://doi.org/10.1016/j.sedgeo.2012.03.019, 2012.

Léonide, P., Fournier, F., Reijmer, J. J. G., Vonhof, H., Borgomano, J., Dijk, J., Rosenthal, M., Van Goethem, M., Cochard, J., and Meulenaars, K.: Diagenetic patterns and pore space distribution along a platform to outer-shelf transect (Urgonian limestone, Barremian-Aptian, SE France), Sediment. Geol., 306, 123, https://doi.org/10.1016/j.sedgeo.2014.03.001, 2014.

Long, J. J. and Imber, J.: Geological controls on fault relay zone scaling, J. Struct. Geol., 33, 1790-1800, https://doi.org/10.1016/j.jsg.2011.09.011, 2011.

Lothe, A. E., Gabrielsen, R. H., Hagen, N. B., and Larsen, B. T.: An experimental study of the texture of deformation bands: effects on the porosity and permeability of sandstones (1990), Petrol. Geosci., 8, 195, https://doi.org/10.1144/petgeo.8.3.195, 2002.

Lucia, F. J.: Origin and petrophysics of dolostone pore space, Geom. Petrog. Dolomite Hydrocarb. Reserv. Geol. Soc. Lond., 235, 141-155, https://doi.org/10.1144/GSL.SP.2004.235.01.06, 2004.

Machel, H. G.: Concepts and models of dolomitization: a critical reappraisal, Geol. Soc. Lond., 235, 7-63, https://doi.org/10.1144/GSL.SP.2004.235.01.02, 2004.

Machel, H. G., Cavell, P. A., Buschkuehle, B. E., and Michael, K.: Tectonically induced fluid flow in Devonian carbonate aquifers of the Western Canada Sedimentary Basin, J. Geochem. Explor., 70, 213-217, https://doi.org/10.1016/S0375-6742(00)00093-5, 2000.

Main, I. G., Kwon, O., Ngwenya, B. T., and Elphick, S. G.: Fault sealing during deformation-band growth in porous sandstone, Geology, 28, 1131-1134, https://doi.org/10.1130/00917613(2000)28<1131:FSDDGI>2.0.CO;2, 2000.

Masse, J.-P. and Philip, J.: Paléogéographie et tectonique du Crétacé moyen en Provence: révision du concept d'isthme durancien, Rev. Géographie Phys. Géologie Dyn., 18, 49-46, 1976.

Masse, J. P.: Les calcaires urgoniens de Provence (ValanginienAptien Inférieur) - Stratigraphie, paléontologie, paléoenvironnements et leur évolution, Marseille, Thèse de la Faculté des Sciences de Luminy (U2), 445 pp., 1976.

Masse, J. P. and Fenerci-Masse, M.: Carbonate production by rudist bivalves. The record of Late Barremian requieniid communities from Provence (SE France), Palaeogeogr. Palaeocl., 234, 239257, https://doi.org/10.1016/j.palaeo.2005.10.010, 2006.

Masse, J. P. and Fenerci Masse, M.: Drowning discontinuities and stratigraphic correlation in platform carbonates, The late Barremian-early Aptian record of southeast France, Cretac. Res., 32, 659-684, https://doi.org/10.1016/j.cretres.2011.04.003, 2011.

Matonti, C., Lamarche, J., Guglielmi, Y., and Marié, L.: Structural and petrophysical characterization of mixed conduit/seal fault zones in carbonates: Example from the Castellas fault (SE France), J. Struct. Geol., 39, 103-121, https://doi.org/10.1016/j.jsg.2012.03.003, 2012.
Micarelli, L., Benedicto, A., and Wibberley, C. A. J.: Structural evolution and permeability of normal fault zones in highly porous carbonate rocks, J. Struct. Geol., 28, 1214-1227, https://doi.org/10.1016/j.jsg.2006.03.036, 2006.

Molli, G., Cortecci, G., Vaselli, L., Ottria, G., Cortopassi, A., Dinelli, E., Mussi, M., and Barbieri, M.: Fault zone structure and fluid-rock interaction of a high angle normal fault in Carrara marble (NW Tuscany, Italy), J. Struct. Geol., 32, 1334-1348, https://doi.org/10.1016/j.jsg.2009.04.021, 2010.

Molliex, S., Bellier, O., Terrier, M., Lamarche, J., Martelet, G., and Espurt, N.: Tectonic and sedimentary inheritance on the structural framework of Provence (SE France): Importance of the Salon-Cavaillon fault, Tectonophysics, 501, 1-16, https://doi.org/10.1016/j.tecto.2010.09.008, 2011.

Moss, S. and Tucker, M. E.: Diagenesis of BarremianAptian platform carbonates (the Urgonian Limestone Formation of SE France): near-surface and shallow-burial diagenesis, Sedimentology, 42, 853-874, https://doi.org/10.1111/j.13653091.1995.tb00414.x, 1995.

Mozley, P. S. and Goodwin, L. B.: Patterns of cementation along a Cenozoic normal fault: a record of paleoflow orientations, Geology, 23, 539-542, https://doi.org/10.1130/00917613(1995)02<0539:POCAAC >2.3.CO;2, 1995.

Nelson, R.: Geologic Analysis of Naturally Fractured Reservoirs, second ed., Gulf Professional Publishing, Boston, 2001.

Ostwald, W.: Lehrbuch der allgemeinen Chemie, Verlag von Wilhelm Engelmann, Leipzig, 2, 909, 1886.

Philip, J.: Les formations calcaires à rudistes du Crétacé supérieur provençal et rhodanien, Thèse de Doctorat, Université de Provence (Marseille), 438 pp., 1970.

Pichon, X. Le, Rangin, C., Hamon, Y., Loget, N., Lin, J. Y., Andreani, L., and Flotte, N.: Geodynamics of the france southeast basin, Bull. la Soc. Geol. Fr., 181, 477-501, https://doi.org/10.2113/gssgfbull.181.6.477, 2010.

Purser, B. H.: Sédimentation et diagenèse des carbonates néritiques récents, Les éléments de la sédimentation et de la diagenèse, Ed. Tech., 1, 366, 1980.

Reches, Z. and Dewers, T. A.: Gouge formation by dynamic pulverization during earthquake rupture, Earth Planet. Sc. Lett., 235, 361-374, https://doi.org/10.1016/j.epsl.2005.04.009, 2005.

Reid, R. P. and Macintyre, I. G.: Microboring Versus Recrystallization: Further Insight into the Micritization Process, J. Sediment. Res., 70, 24-28, https://doi.org/10.1306/2DC408FA0E47-11D7-8643000102C1865D, 2000.

Roche, V.: Analyse structurale et géo-mécanique de réseau de failles du chaînon de La Fare les Oliviers (Provence), Univ. Montpellier 2, 45, 2008.

Rossetti, F., Aldega, L., Tecce, F., Balsamo, F., Billi, A., and Brilli, M.: Fluid flow within the damage zone of the Boccheggiano extensional fault (Larderello-Travale geothermal field, central Italy): Structures, alteration and implications for hydrothermal mineralization in extensional settings, Geol. Manage., 148, 558579, https://doi.org/10.1017/S001675681000097X, 2011.

Saller, A. H. and Henderson, N.: Distribution of Porosity and Permeability in Platform Dolomites: Insight from the Permian of West Texas: reply, Am. Assoc. Pet. Geol. Bull., 85, 530-532, https://doi.org/10.1306/090800850530, 2001.

Sallier, B.: Carbonates microporeux: influence de l'architecture du milieu poreux et de la mouillabilité sur les écoulements 
diphasiques dans les réservoirs pétroliers, Univ. Genève, 230 pp., 2005.

Samankassou, E., Tresch, J., and Strasser, A.: Origin of peloids in Early Cretaceous deposits, Dorset, South England, Facies, 51, 264-273, https://doi.org/10.1007/s10347-005-0002-8, 2005.

Séranne, M.: The Gulf of Lion continental margin (NW Mediterranean) revisited by IBS: an overview, Geol. Soc. Lond., 156, 15-36, https://doi.org/10.1144/GSL.SP.1999.156.01.03, 1999.

Sibley, D. F. and Gregg, J. A. Y. M.: Classification of Dolomite Rock Texture, J. Sediment. Petrol., 57, 967975, https://doi.org/10.1306/212F8CBA-2B24-11D78648000102C1865D, 1987.

Sibson, R. H.: Crustal stress, faulting and fluid flow, Geol. Soc. Lond., 78, 69-84, https://doi.org/10.1144/GSL.SP.1994.078.01.07, 1994.

Sibson, R. H.: Structural permeability of fluid-driven fault-fracture meshes, J. Struct. Geol., 18, 1031-1042, https://doi.org/10.1016/0191-8141(96)00032-6, 1996.

Sinisi, R., Petrullo, A. V., Agosta, F., Paternoster, M., Belviso, C., and Grassa, F.: Contrasting fault fluids along high-angle faults: a case study from Southern Apennines (Italy), Tectonophysics, 690, 206-218, https://doi.org/10.1016/j.tecto.2016.07.023, 2016.

Solum, J. G. and Huisman, B. A. H.: Toward the creation of models to predict static and dynamic fault-seal potential in carbonates, Pet. Geosci., 23, 70-91, https://doi.org/10.1144/petgeo2016-044, 2016.

Solum, J. G., Davatzes, N. C., and Lockner, D. A.: Fault-related clay authigenesis along the Moab Fault: Implications for calculations of fault rock composition and mechanical and hydrologic fault zone properties, J. Struct. Geol., 32, 1899-1911, https://doi.org/10.1016/j.jsg.2010.07.009, 2010.

Storti, F., Billi, A., and Salvini, F.: Particle size distributions in natural carbonate fault rocks: Insights for non-selfsimilar cataclasis, Earth Planet. Sc. Lett., 206, 173-186, https://doi.org/10.1016/S0012-821X(02)01077-4, 2003.

Swart, P. K.: The geochemistry of carbonate diagenesis: The past, present and future, Sedimentology, 62, 1233-1304, https://doi.org/10.1111/sed.12205, 2015.

Tempier, C.: Modèle nouveau de mise en place des structures provençales, Bull. la Soc. Geol. Fr., 3, 533-540, https://doi.org/10.2113/gssgfbull.III.3.533, 1987.

Tondi, E.: Nucleation, development and petrophysical properties of faults in carbonate grainstones: Evidence from the San Vito Lo Capo peninsula (Sicily, Italy), J. Struct. Geol., 29, 614-628, https://doi.org/10.1016/j.jsg.2006.11.006, 2007.
Triat, J.: Paléoaltérations dans le crétacé supérieur de Provence rhodanienne, Strasbourg: Institut de Géologie - Université LouisPasteur., 223 pp., 1982.

Vincent, B., Emmanuel, L., Houel, P., and Loreau, J. P.: Geodynamic control on carbonate diagenesis: Petrographic and isotopic investigation of the Upper Jurassic formations of the Paris Basin (France), Sediment. Geol., 197, 267-289, https://doi.org/10.1016/j.sedgeo.2006.10.008, 2007.

Volery, C., Davaud, E., Foubert, A., and Caline, B.: Shallowmarine microporous carbonatereservoir rocks in the Middle East: relationship with seawater $\mathrm{Mg} / \mathrm{Ca}$ ration and eustatic sea level, J. Pet. Geol., 32, 313-325, https://doi.org/10.1111/j.17475457.2009.00452.x, 2009.

Volery, C., Davaud, E., Foubert, A., and Caline, B.: Lacustrine microporous micrites of the Madrid Basin (Late Miocene, Spain) as analogues for shallow-marine carbonates of the Mishrif reservoir formation (Cenomanian to Early Turonian, Middle East), Facies, 56, 385-397, https://doi.org/10.1007/s10347-009-0210-8, 2010.

Walsh, J. J., Watterson, J., Bailey, W. R., and Childs, C.: Fault relays, bends and branch-lines, 21, 1019-1026, https://doi.org/10.1016/S0191-8141(99)00026-7, 1999.

Walsh, J. J., Bailey, W. R., Childs, C., Nicol, A., and Bonson, C. G.: Formation of segmented normal faults: a 3-D perspective, 25, 1251-1262, https://doi.org/10.1016/S0191-8141(02)00161X, 2003.

Wilkins, S. J., Naruk, S. J., Wilkins, S. J., International, S., Naruk, S. J., and International, S.: Quantitative analysis of slip-induced dilation with application to fault seal, AAPG Bulletin, 1, 97-113, https://doi.org/10.1306/08010605177, 2007.

Woodcock, N. H., Dickson, J. A. D., and Tarasewicz, J. P. T.: Transient permeability and reseal hardening in fault zones: evidence from dilation breccia textures, Geol. Soc. Lond., 270, 43-53, 2007.

Wu, G., Gao, L., Zhang, Y., Ning, C., and Xie, E.: Fracture attributes in reservoir-scale carbonate fault damage zones and implications for damage zone width and growth in the deep subsurface, J. Struct. Geol., 118, 181-193, https://doi.org/10.1016/j.jsg.2018.10.008, 2019.

Zhang, Y., Schaubs, P. M., Zhao, C., Ord, A., Hobbs, B. E., and Barnicoat, A. C.: Fault-related dilation, permeability enhancement, fluid flow and mineral precipitation patterns: numerical models, Geol. Soc. Lond., 299, 239-255, https://doi.org/10.1144/SP299.15, 2008.

Zhu, W. and Wong, T.-F.: The transition from brittle faulting to cataclastic flow: Permeability evolution, J. Geophys. Res., 102, 3027-3041, https://doi.org/10.1029/96JB03282, 1997. 